\title{
Decomposing Berge graphs containing no proper wheel, long prism or their complements
}

\author{
Michele Conforti * \\ Gérard Cornuéjols ${ }^{\dagger}$ \\ Giacomo Zambelli $\ddagger$
}

May 2002, revised November 2003

\begin{abstract}
In this paper we show that, if $G$ is a Berge graph such that neither $G$ nor its complement $\bar{G}$ contains certain induced subgraphs, named proper wheels and long prisms, then either $G$ is a basic perfect graph (a bipartite graph, a line graph of a bipartite graph or the complement of such graphs) or it has a skew partition that cannot occur in a minimally imperfect graph. This structural result implies that $G$ is perfect.
\end{abstract}

\section{Introduction}

A graph is perfect if, in all its induced subgraphs, the size of a largest clique is equal to the chromatic number. A graph is Berge if it does not contain an odd hole or its complement. The strong perfect graph conjecture (SPGC) [1] states that Berge graphs are perfect. In 2001, Conforti, Cornuéjols and Vušković [6] suggested the following approach to solving the SPGC: show that all Berge graphs can be decomposed into four basic classes of perfect graphs (bipartite graphs, line graphs of bipartite graphs and their complements) using decompositions that cannot occur in minimally imperfect graphs (2-joins and certain kinds of skew partitions). Chudnovsky, Robertson, Seymour, Thomas [3] announced recently that they solved the SPGC using this approach. Conforti, Cornuéjols, Vušković, Zambelli obtained partial results discussed in the present paper and in [7]. There are

\footnotetext{
${ }^{*}$ Dipartimento di Matematica Pura ed Applicata, Università di Padova, Via Belzoni 7, 35131 Padova, Italy. conforti@math.unipd.it

${ }^{\dagger}$ GSIA, Carnegie Mellon University, Schenley Park, Pittsburgh, PA 15213, USA; and LIF, University of Marseille, Luminy, France. gc0v@andrew.cmu.edu

${ }^{\ddagger}$ GSIA, Carnegie Mellon University, Schenley Park, Pittsburgh, PA 15213, USA. giacomo@andrew.cmu.edu This work was supported in part by NSF grant DMI-0098427 and ONR grant N00014-97-1-0196.
} 
three separate cases to decomposing Berge graphs $G$ depending on whether they contain certain induced subgraphs called long prisms and proper wheels (to be defined later):

(i) Neither $G$ nor its complement $\bar{G}$ contains a long prism or a proper wheel,

(ii) $G$ contains a long prism,

(iii) $G$ contains a proper wheel but neither $G$ nor $\bar{G}$ contains a long prism.

This paper proves a decomposition theorem for Berge graphs that satisfy (i). Chudnovsky, Robertson, Seymour, Thomas [3] simultaneously proved a similar result. Furthermore, they obtained a decomposition of Berge graphs that satisfy (ii). Finally they also showed in [3] that no minimally imperfect Berge graph satisfies (iii), thus solving the SPGC. Our results on decomposing Berge graphs that satisfy (iii) are available in [7].

\subsection{Notation and definitions}

We refer to West [13] for standard terminology in graph theory. Let $G$ be an undirected simple graph. We denote by $\bar{G}$ the complement graph of $G$. Given $X \subset V(G)$, we denote by $G[X]$ the subgraph of $G$ induced by $X$ and by $\bar{G}[X]$ the subgraph of $\bar{G}$ induced by $X$. Given two distinct nodes $x, y \in V(G)$ and an induced subgraph $G^{\prime}$ of $G$, we say that $x$ and $y$ are twins with respect to $G^{\prime}$ if they have the same neighbors in $G^{\prime} \backslash\{x, y\}$. If $x$ and $y$ are adjacent (resp. not adjacent) we say that $x$ and $y$ are true twins (resp. false twins) with respect to $G^{\prime}$.

Given a path $P=x_{1}, \ldots, x_{n}$ and $1 \leq i \leq j \leq n$, we denote with $P_{x_{i} x_{j}}$ the path $x_{i}, \ldots, x_{j}$ contained in $P$, and we say that $P_{x_{i} x_{j}}$ is a sub-path of $P$. The set $\left\{x_{i} \mid 1<i<n\right\}$ is the interior of $P$. The nodes in the interior of $P$ are also referred to as the intermediate nodes of $P$. The length of a path is its number of edges, and it is denoted by $|P|$. $P$ is said to be odd (resp. even) if $P$ has odd (resp. even) length.

A path in $\bar{G}$ is called a co-path. The interior and the length of a co-path $P$ are, respectively, the interior and the length of $\bar{P}$. A set $X \subseteq V(G)$ is said to be co-connected if $\bar{G}[X]$ is a connected graph.

Given a set $X \subset V(G)$, a node $x \notin X$ and an edge $e=y z$ such that $y, z \notin X$, we say that $x$ is universal for $X$ if $x$ is adjacent to every node of $X$, and we say that $e$ sees $X$ if both $y$ and $z$ are universal for $X$.

Given two disjoint sets of nodes $A$ and $B$, a direct connection between $A$ and $B$ is a minimal chordless path (in term of its node set) $P=x_{1}, \ldots, x_{n}$ such that $x_{1}$ has a neighbor in $A$ and $x_{n}$ has a neighbor in $B$.

Definition 1 Given two node disjoint triangles $a_{1}, a_{2}, a_{3}$ and $b_{1}, b_{2}, b_{3}$, a long prism is a graph induced by three node disjoint chordless paths $P^{1}=a_{1}, \ldots, b_{1}, P^{2}=a_{2}, \ldots, b_{2}$ and $P^{3}=a_{3}, \ldots, b_{3}$, at least one of which has length greater than one, such that the only adjacencies between the nodes of distinct paths are the edges of the two triangles. We denote such a graph by $3 P C\left(a_{1} a_{2} a_{3}, b_{1} b_{2} b_{3}\right)$. 
The following concepts were introduced in [5].

Definition $2 A$ wheel $(H, v)$ consists of a hole $H$ and a node $v$ not in $H$ that has at least 3 neighbors in $H$. If $v$ has $k$ neighbors in $H$, we say that $(H, v)$ is a $k$-wheel. The node $v$ is called the center of the wheel. If $x$ and $y$ are neighbors of $v$ in $H$, a sub-path of $H$ with endnodes $x$ and $y$ is called $a$ sector if it contains no neighbor of $v$ in its interior.

An odd wheel is a wheel containing an odd number of triangles. An even wheel is a wheel which is not odd.

A triangle-free wheel is a wheel containing no triangle.

$A$ twin wheel is a 3-wheel $(H, v)$ such that $(H, v)$ contains exactly 2 triangles.

$A$ line wheel is a 4-wheel $(H, v)$ such that $(H, v)$ contains exactly 2 triangles and these triangles have only node $v$ in common.

$A$ universal wheel is a wheel $(H, v)$ in which $v$ is adjacent to every node of $H$.

A proper wheel is an even wheel which is not a triangle-free wheel, a twin wheel, a line wheel or a universal wheel.

A double line wheel $(H, u, v)$ is the graph induced by a hole $H$ and two distinct nonadjacent nodes $u$ and $v$ not in $H$ such that $(H, u)$ and $(H, v)$ are both line wheels and the edges of $H$ that see $u$ or $v$ are distinct and these four edges alternate between those that see $u$ and those that see $v$.

Definition $3 A$ cap $(H, v)$ consists of a hole $H$ and a node $v$ not in $H$ that has exactly two neighbors $a$ and $b$ in $H$, and $a$ and $b$ are adjacent. We say that $v$ is the tip of $(H, v)$ while $a$ and $b$ are the attachments of $v$ in $H$.

A hole or an anti-hole is big if it has at least 6 nodes. If $(H, v)$ is a wheel or a cap, we say that $(H, v)$ is big if $H$ is big.

A graph $G$ has a skew partition if the nodes of $V(G)$ can be partitioned into nonempty sets $A, B, C, D$ such that every node of $A$ is adjacent to every node of $B$ and there is no edge between $C$ and $D$. We say that the set $A \cup B$ is a skew cutset of $G$ that separates $C$ from $D$. When $A$ or $B$ has cardinality one, $A \cup B$ is called a star cutset. Chvátal [4] conjectured that a minimally imperfect graph cannot have a skew partition. Recently, Chudnovsky, Robertson, Seymour and Thomas [3] proved the conjecture. Previously, Robertson, Seymour and Thomas [11] had shown that the skew-partition conjecture holds for special types of skew partitions. Namely, a skew partition $(A, B, C, D)$ is good if either $C$ contains a node universal for $A$, or $A$ contains a node with no neighbors in $C$. For example, a star cutset defines a good skew partition.

Theorem 4 (Robertson, Seymour and Thomas [11]) No minimal imperfect graph contains a good skew partition.

Theorem 4 generalizes previous results due to Hoàng, who showed that no minimally imperfect graph contains a T-cutset (i.e. a skew cutset in which both $C$ and $D$ contain a node universal for $A$ ) or a U-cutset (i.e. a skew cutset in which $C$ contains a node universal for $A$ and a node universal for $B$ ). 
A Berge graph is basic if it belongs to one of the following four classes of perfect graphs: bipartite graphs, line graphs of bipartite graphs and their complements.

In this paper we will prove the following.

Theorem 5 Let $G$ be a Berge graph such that neither $G$ nor $\bar{G}$ contains a proper wheel or a long prism. Then either $G$ is basic, or $G$ contains a good skew partition.

Note that, since basic graphs are perfect, Theorems 4 and 5 imply that the Strong Perfect Graph Conjecture holds for graphs containing no proper wheel, long prism or their complements.

To prove Theorem 5, we will sometimes use the following lemma, due to Roussel and Rubio [12] and proved independently also by Robertson, Seymour and Thomas [11], who divulged it as a useful tool in the study of Berge graphs and named it The Wonderful Lemma.

Lemma 6 (The Wonderful Lemma) (Roussel and Rubio [12]) Let G be a Berge graph where $V(G)$ can be partitioned into a co-connected set $S$ and an odd chordless path $P=u, u^{\prime}, \ldots, v^{\prime}, v$ of length at least 3 such that $u$ and $v$ are both universal for $S$. Then one of the following holds:

(i) An odd number of edges of $P$ see $S$.

(ii) $|P|=3$ and $S \cup\left\{u^{\prime}, v^{\prime}\right\}$ contains an odd chordless co-path between $u^{\prime}$ and $v^{\prime}$.

(iii) $|P| \geq 5$ and there exist two nonadjacent nodes $x, x^{\prime}$ in $S$ such that $(V(P) \backslash\{u, v\}) \cup$ $\left\{x, x^{\prime}\right\}$ induces a chordless path.

The original proof of the Wonderful Lemma (with a slightly different statement) can be found in [12]. A similar proof is contained in [7].

Remark 7 In a Berge graph containing no long prism, only the first two outcomes of the Wonderful Lemma are possible. Indeed, if $P$ has length at least 5 and there exist two nonadjacent nodes $x, x^{\prime}$ in $S$ such that $(V(P) \backslash\{u, v\}) \cup\left\{x, x^{\prime}\right\}$ induces a chordless path, then $V(P) \cup\left\{x, x^{\prime}\right\}$ induces a long prism.

We will also use the fact that the following structure contains an odd hole and therefore cannot occur in a Berge graph. Given a triangle $a_{1}, a_{2}, a_{3}$ and a node $y$ distinct from $a_{1}, a_{2}, a_{3}$, a $3 P C\left(a_{1} a_{2} a_{3}, y\right)$ is a graph induced by three chordless paths $P^{1}=a_{1}, \ldots, y$, $P^{2}=a_{2}, \ldots, y$ and $P^{3}=a_{3}, \ldots, y$, having no common nodes other than $y$ and such that the only adjacencies between nodes of $P^{i} \backslash y$ and $P^{j} \backslash y$, for $i, j \in\{1,2,3\}$ distinct, are the edges of the triangle. Also, at most one of the paths $P^{1}, P^{2}, P^{3}$ is an edge.

\section{Proof of Theorem 5}

Let $G$ be a Berge graph such that neither $G$ nor $\bar{G}$ contains a proper wheel or a long prism. 


\subsection{Double line wheels}

Lemma 8 If $G$ contains a double line wheel $(H, u, v)$, then either $G$ is the line graph of a bipartite graph or it contains a good skew partition.

Proof: If $|H|>6$, let $a b$ and $c d$ be the edges of $H$ that see $u$ and assume, by symmetry, that $a$ and $c$ are endnodes of a sector, denoted by $H_{a c}$, of length at least 4 . Let $s t$ be the edge of $H_{a c}$ that sees $v$. W.l.o.g. $a \neq s, t$. Then $G$ contains a long prism $3 P C(u a b, s t v)$.

Hence $|H|=6$. Let $H=\left(a_{1}, \ldots, a_{6}, a_{1}\right)$ and assume that $u$ is adjacent to $a_{1}, a_{2}$, $a_{4}$ and $a_{5}$ and that $v$ is adjacent to $a_{2}, a_{3}, a_{5}$ and $a_{6}$. Let $H^{\prime}=\left(a_{1}, u, a_{4}, a_{3}, v, a_{6}, a_{1}\right)$, $Q=\left(a_{1}, v, u, a_{6}, a_{2}, a_{5}, a_{1}\right)$ and $Q^{\prime}=\left(a_{2}, a_{5}, a_{3}, u, v, a_{4}, a_{2}\right)$. Then $H^{\prime}$ is a 6 -hole and $\left(H^{\prime}, a_{2}, a_{5}\right)$ is a double line wheel, while $Q$ and $Q^{\prime}$ are both 6 -anti-holes and $\left(Q, a_{3}, a_{4}\right)$ $\left(Q^{\prime}, a_{1}, a_{6}\right)$ are both double line wheels in $\bar{G}$.

For $x \in V \backslash(V(H) \cup\{u, v\})$, we examine the adjacencies between $x$ and $(H, u, v)$. Since, as we just observed, the complement of a double line wheel is a double line wheel, then, by going to the complement, we can assume that $x$ has at most four neighbors in $(H, u, v)$.

Claim 1: If $x$ has at most four neighbors in $(H, u, v)$, then one of the following holds, up to the symmetries of $(H, u, v)$ :

(i) $x$ has no neighbor in $(H, u, v)$;

(ii) $x$ is true or false twin of one of the nodes in $(H, u, v)$ w.r.t. $(H, u, v)$;

(iii) The only neighbors of $x$ in $(H, u, v)$ are $a_{1}, a_{3}, a_{4}$ and $a_{6}$;

(iv) The only neighbors of $x$ in $(H, u, v)$ are $a_{1}$ and $a_{6}$;

(v) The only neighbors of $x$ in $(H, u, v)$ are $a_{1}, a_{2}$ and $u$.

Proof of Claim 1: Since $G$ does not contain any proper wheel, then $(H, x)$ can be a universal wheel, a twin wheel, a line wheel or $V(H) \cup x$ induces a triangle-free-graph or a cap. If $(H, x)$ is a universal wheel, then $x$ has more than four neighbors in $(H, u, v)$. Assume that $(H, x)$ is a twin wheel and let $N_{H}(x)=\left\{a_{i-1}, a_{i}, a_{i+1}\right\}$. Then $x$ is adjacent to $u$ if and only if $a_{i}$ is a neighbor of $u$, otherwise, if $C$ is the hole obtained from $H$ by replacing $a_{i}$ with $x$, then $(C, u)$ is a proper wheel or an odd wheel. Similarly, $x$ is adjacent to $v$ if and only if $a_{i}$ is a neighbor of $v$. But then $x$ is a twin of $a_{i}$ w.r.t. $(H, u, v)$. Assume now that $(H, x)$ is a line wheel. Since $x$ has already four neighbors in $(H, u, v)$, either $x$ is a false twin of $u$ or $v$ or (iv) holds.

Assume next that $V(H) \cup x$ induces a cap. By symmetry, we can assume that $x$ is adjacent to $a_{1}$. Assume first that $x$ is adjacent to $a_{1}$ and $a_{6}$. If $x$ is adjacent to both $u$, $v$, then $\left(Q^{\prime}, x\right)$ is an odd wheel in $\bar{G}$. If $x$ is adjacent to exactly one of $u, v$, say $u$, then $x, u, a_{2}, v, a_{6}$ induces a 5 -hole. So (iv) must hold. Assume now that $x$ is adjacent to $a_{1}$ and $a_{2}$. Then (v) must hold since, otherwise, $\left(Q^{\prime}, x\right)$ is a proper wheel in $\bar{G}$.

Finally, assume that $V(H) \cup x$ induces a triangle-free graph. By symmetry we can assume that $V\left(H^{\prime}\right) \cup x$ also induces a triangle-free graph. If $x$ has no neighbor in $(H, u, v)$, (i) holds. Thus, by symmetry, we can assume that $x$ is either adjacent to $a_{1}$ or to $a_{2}$. If $x$ is 
adjacent to $a_{1}$, then $x$ is not adjacent to $a_{2}, a_{6}$ and $u$, and $(Q, x)$ must be a twin wheel in $\bar{G}$, hence $x$ is a twin of $a_{6}$ w.r.t. $(H, u, v)$. Assume then that $x$ is adjacent to $a_{2}$ and, by symmetry and by the previous case, assume $x$ is not adjacent to $a_{1}, a_{3}, a_{4}$ and $a_{6}$. Also, $x$ is not adjacent to $a_{5}$, else there is a 5 -hole $\left(a_{1}, a_{2}, x, a_{5}, a_{6}, a_{1}\right)$. Hence $(Q, x)$ must be a line wheel in $\bar{G}$, so $x$ is adjacent to $v$ but not to $u$, but now $\left(Q^{\prime}, x\right)$ is a proper wheel in $\bar{G}$. This completes the proof of Claim 1 .

We say that a graph $G^{\prime}$ is an extended multi line wheel if $G^{\prime}$ can be partitioned into sets $A_{1}, \ldots, A_{6}, U, V$ and $W$ with the property that every node in $A_{i}$ is adjacent to every node in $A_{i+1}$ (where the indices are taken modulo 6), every node in $U$ (resp. $V$ ) (resp. $W$ ) is adjacent to every node in $A_{1}, A_{2}, A_{4}$ and $A_{5}$ (resp. $\left.A_{2}, A_{3}, A_{5}, A_{6}\right)$ (resp. $A_{1}, A_{3}$, $\left.A_{4}, A_{6}\right)$ and these are the only edges with endnodes in different sets of the partition. All the sets, except at most $W$, are nonempty.

Since $G$ contains a double line wheel $(H, u, v)$, then $G$ contains an extended multi line wheel $G^{\prime}$ such that $a_{i} \in A_{i}, u \in U$ and $v \in V$. Assume $G^{\prime}$ is maximal (in terms of its node set) with this property.

\section{Claim 2:}

- Every node of Type (1)(iii) w.r.t. $(H, u, v)$ belongs to $W$.

- If a node $x$ of Type (1)(ii) w.r.t. $(H, u, v)$ does not belongs to $G^{\prime}$, then $x$ is a true twin of a node of degree 3 in $(H, u, v)$, say $a_{1}$, and $x$ is of Type $(v)$ w.r.t. $\left(H^{*}, u, v\right)$ for some 6 -hole $H^{*}$ obtained from $H$ by replacing $a_{6}$ by a node $a_{6}^{*} \in A_{6}$.

- If a node $x$ is of Type (1)(iv) w.r.t. $(H, u, v)$, adjacent to $a_{1}$ and $a_{6}$, then $x$ is universal for $A_{1} \cup A_{6}$ and has no neighbor in $G^{\prime} \backslash A_{1} \cup A_{6}$.

- If a node $x$ is of Type $(1)(\mathrm{v})$ w.r.t. $(H, u, v)$, adjacent to $a_{1}, a_{2}$ and $u$, then $x$ is universal for $A_{1} \cup A_{2} \cup U$ and has no neighbor in $A_{3} \cup A_{4} \cup A_{5} \cup V$.

Proof of Claim 2: By construction, every node of $G^{\prime}$ must be a twin of a node of $(H, u, v)$ w.r.t. $(H, u, v)$ or must be of Type (1)(iii). Suppose that some node $x$ of Type (1)(iii) does not belong to $W$. Then either $x$ is not adjacent to some node $y$ in $A_{1}, A_{3}, A_{4}$ or $A_{6}$ or $x$ is adjacent to some node $y$ in $A_{2}, A_{5}, U$ or $V$. Let $\left(H^{*}, u^{*}, v^{*}\right)$ be the double line wheel obtained by adding $y$ and removing the corresponding node of $(H, u, v)$. Now $x$ contradicts Claim 1 in $\left(H^{*}, u^{*}, v^{*}\right)$ or in its complement. So the first part of Claim 2 holds. Now suppose that some node $x$ of Type (1)(ii) does not belong to $G^{\prime}$. By symmetry we can assume that $x$ is a twin of $a_{1}$ or $a_{2}$ w.r.t. $(H, u, v)$. If $x$ is a twin of $a_{1}$, then either $x$ is not adjacent to some node $y$ in $A_{2}, A_{6}$ or $U$ or $x$ is adjacent to some node $y$ in $A_{3}, A_{4}, A_{5}$ or $V$. Constructing $\left(H^{*}, u^{*}, v^{*}\right)$ as above, we obtain a contradiction of Claim 1 unless $y$ is in $A_{6}$. If $x$ is a twin of $a_{2}$, constructing $\left(H^{*}, u^{*}, v^{*}\right)$ as above, we obtain a contradiction of Claim 1 in all cases. The last two statements of Claim 2 follow similarly. This completes the proof of Claim 2. 
By Claim 2, the nodes of $G \backslash G^{\prime}$ partition into two sets $X$ and $Y$ as follows: $X$ contains the nodes of $G \backslash G^{\prime}$ that have no neighbor in $V\left(G^{\prime}\right)$ or are of Type (1)(iv) or (v) w.r.t.at least one double line wheel $\left(H^{*}, u^{*}, v^{*}\right)$ where $H^{*}=a_{1}^{*}, \ldots, a_{6}^{*}, a_{1}^{*}$ with $a_{i}^{*} \in A_{i}, u^{*} \in U$, $v^{*} \in V$. The set $Y$ contains the remaining nodes of $G \backslash G^{\prime}$. In the complement graph $\bar{G}$, the nodes of $Y$ have either no neighbor in $V\left(G^{\prime}\right)$ or are of Type (1)(iv) or (v) w.r.t. at least one double line wheel $\left(Q^{*}, a_{3}^{*}, a_{4}^{*}\right)$ where $Q^{*}=a_{1}^{*}, v^{*}, u^{*}, a_{6}^{*}, a_{2}^{*}, a_{5}^{*}, a_{1}^{*}$ with $a_{i}^{*} \in A_{i}$, $u^{*} \in U, v^{*} \in V$.

Claim 3: Let $X_{1,2}$ be the set of nodes of $X$ that are universal for $A_{1} \cup A_{2} \cup U$ and possibly adjacent to nodes of $A_{6}$ but to no other nodes of $G^{\prime}$. Then there exists a node of $A_{6}$ that has no neighbor in $X_{1,2}$.

Proof of Claim 3: Suppose not. Since every node of $A_{6}$ has a neighbor in $X_{1,2}$ and every node of $X_{1,2}$ has a non-neighbor in $A_{6}$, there must exist $r, s \in A_{6}$ and $t, z \in X_{1,2}$ such that $r t$ and $s z$ are edges but $r z$ and $s t$ are not. Indeed, it is immediate to verify that the statement is true if $\left|A_{6}\right| \leq 2$ or $\left|X_{1,2}\right| \leq 2$. By induction on $\left|A_{6}\right|+\left|X_{1,2}\right|$, given $z \in X_{1,2}$, either we are done by applying the inductive hypothesis to $A_{6}$ and $X_{1,2} \backslash z$, or $A_{6}$ contains a node $s$ with no neighbors in $X_{1,2} \backslash z$ so $z$ and $s$ are adjacent. Let $r$ be a non-neighbor of $z$ in $A_{6}$ and $t$ be a neighbor of $r$ in $X_{1,2}$, then $r t$ and $s z$ are edges but $r z$ and st are not.

If neither $r s$ nor $z t$ is an edge, consider the 6-hole $L=r, t, a_{2}, z, s, a_{5}, r$. Then $(L, u)$ is a proper wheel. If exactly one of $r s$ and $z t$ is an edge, there is a 5-hole $r, t, a_{2}, z, s, r$ or $r, t, z, s, a_{5}, r$. If both $r s$ and $z t$ are edges, the nodes in $\left\{r, s, t, z, a_{2}, a_{3}, a_{4}, a_{5}\right\}$ induce a long prism. This proves Claim 3.

Claim 4: If $x_{i}, x_{j} \in X$ are universal for $A_{i}, A_{i+1}$ and for $A_{j}, A_{j+1}$, respectively, where $1 \leq i<j \leq 5$, and possibly to other nodes of $G^{\prime}$, then $x_{i}$ and $x_{j}$ are in different connected components of $G[X]$.

Proof of Claim 4: Suppose not. Choose a pair $x_{i}, x_{j} \in X, i<j$, with a shortest path $P$ connecting them in $G[X]$. By the choice of $P$, the internal nodes of $P$ have no neighbor in $G^{\prime}$. By Claim 2, there exists a double line wheel $\left(H^{*}, u^{*}, v^{*}\right)$ where $H^{*}=a_{1}^{*}, \ldots, a_{6}^{*}, a_{1}^{*}$ with $a_{i}^{*} \in A_{i}, u^{*} \in U, v^{*} \in V$, such that $x_{i}$ and $x_{j}$ are both of Type (1)(iv) or (v) w.r.t. $\left(H^{*}, u^{*}, v^{*}\right)$. If $j-i \geq 2$, the nodes of $H \cup P$ induce a long prism. If $j=i+1$, it is sufficient to consider the cases $j=2$ and $j=3$ by symmetry. If $j=2$, the nodes of $V(P) \cup\left\{a_{2}, a_{3}, a_{4}, a_{5}, u, v\right\}$ induce a long prism. If $j=3$, the nodes of $V(P) \cup\left\{a_{2}, a_{4}, a_{5}, u, v\right\}$ induce a long prism. This proves Claim 4 .

Assume that $Y$ is nonempty. By symmetry $Y$ contains a node $y$ universal for $A_{2} \cup$ $A_{3} \cup A_{5} \cup A_{6}$. Furthermore, if $y$ is of Type (1)(iv) in $\bar{G}$, we can assume that, in $G, y$ is universal for $A_{1} \cup A_{4}$ and has no neighbor in $U \cup V$. If $y$ is of Type (1)(v) in $\bar{G}$, we can assume that, in $G, y$ has no neighbor in $A_{1} \cup A_{4} \cup V$. Finally, if all the nodes of $Y$ are universal for $G^{\prime}$, choose $y$ to be any node of $Y$. Let $A$ be the co-connected component of $Y$ containing $y$ and let $B$ be the set of nodes $A_{2} \cup A_{3} \cup A_{5} \cup A_{6}$ together with the nodes of $G \backslash G^{\prime}$ that are universal for $A$. By Claim 4 applied to $\bar{G}, A$ is universal for 
$A_{2} \cup A_{3} \cup A_{5} \cup A_{6}$. Clearly, $A$ is universal for $Y \backslash A$. Therefore, by Claim 4 applied to $G, A \cup B$ is a skew cutset separating $V$ from $A_{1} \cup A_{4} \cup U$. By Claim 3 applied to $\bar{G}$, if $y$ is of Type $(1)(\mathrm{v})$ in $\bar{G}$, at least one node of $U$ is universal for $A$. And if $y$ is not of Type (1)(v) in $\bar{G}$, the nodes of $A_{1}$ are universal for $A$. So $A \cup B$ is a good skew cutset.

By the argument above applied to $\bar{G}$, if $X$ is nonempty then $G$ has a good skew partition. Hence we may assume that $X$ and $Y$ are both empty. If any of the sets $A_{1}, \ldots, A_{6}, U, V, W$ has cardinality greater than one, then $G$ has a star cutset. So, if $G$ has no good skew partition, $G^{\prime}$ is a multi line wheel.

\subsection{Line wheels}

Lemma 9 If $G$ contains a line wheel, then $G$ is either the line graph of a bipartite graph, or it contains a good skew partition.

Proof: By Lemma 8, if $G$ contains a double line wheel then we are done. Hence we can assume that $G$ does not contain a double line wheel or its complement.

Assume that $G$ contains a line wheel $(H, v)$. Let $a b$ and $c d$ be the two edges of $H$ that see $v$. Assume, w.l.o.g., that $a$ and $c$ are the endpoints of a sector of $(H, v)$, denoted by $H_{a c}$ while $b$ and $d$ are the endpoints of the other sector of $(H, v)$, denoted by $H_{b d}$. Let $A$ be a maximal co-connected set in $G \backslash H$ such that $v \in A$ and $A$ sees both $a b$ and $c d$ (note that the nodes in $A$ are either centers of line wheels w.r.t. $H$ or they are universal for $H)$. Let $B$ be the set of nodes that are universal for $A$ in $G \backslash(A \cup\{b, c\})$. If $H_{a c} \backslash a$ and $H_{b d} \backslash d$ lie in distinct connected components of $G \backslash(A \cup B)$, then let $C$ be the connected component containing $H_{a c} \backslash a$ and $D=V(G) \backslash(A \cup B \cup C)$. Then $(A, B, C, D)$ is a skew partition, $c \in C$ and $b \in D$ are both universal for $A$, hence $A \cup B$ is a T-cutset and we are done.

We will show that it must be the case that $H_{a c} \backslash a$ and $H_{b d} \backslash d$ lie in distinct connected components of $G \backslash(A \cup B)$. Assume not. Then there exists a path $P=x_{1}, \ldots, x_{n}$ in $G \backslash(V(H) \cup A \cup B)$ such that $x_{1}$ has a neighbor in $H_{a c} \backslash a, x_{n}$ has a neighbor in $H_{b d} \backslash d$ and no intermediate node of $P$ has a neighbor in $H \backslash\{a, d\}$.

Claim 1: $a$ and $d$ cannot both have a neighbor in $P \backslash\left\{x_{1}, x_{n}\right\}$.

Proof of Claim 1: Assume not and let $x_{i}$ and $x_{j}(2 \leq i, j \leq n-1)$ be two nodes at minimum distance in $P$ such that $x_{i}$ is adjacent to $a$ and $x_{j}$ is adjacent to $d$. Then $F=a, x_{i}, P_{x_{i} x_{j}}, x_{j}, d$ is an odd path, otherwise $C=\left(a, F, d, c, H_{a c}, a\right)$ would be an odd hole. Since $a$ and $d$ are both universal for $A$ and no node in $F \backslash\{a, d\}$ is universal for $A$, then, by Lemma 6 and Remark 7, $F$ has length 3 and there exists an odd chordless co-path $Q$ between $x_{i}$ and $x_{j}$ contained in $A \cup\left\{x_{i}, x_{j}\right\}$. Thus $C=\left(b, x_{i}, Q, x_{j}, b\right)$ is an odd anti-hole, a contradiction. This completes the proof of Claim 1.

Claim 2: No node of $P$ has a neighbor in both $H_{a c}$ and $H_{b d}$.

Proof of Claim 2: By contradiction, assume $x_{i}(1 \leq i \leq n)$ has a neighbor in both $H_{a c}$ and $H_{b d}$.

Case 1: $x_{i}$ has two nonadjacent neighbors in $H$. 
Then $\left(H, x_{i}\right)$ must be either a universal wheel, a twin wheel, a line wheel or $H \cup x_{i}$ is a triangle-free graph. If $x_{i}$ sees both $a b$ and $c d$, then, since $x_{i}$ is not universal for $A$, $A \cup x_{i}$ is a co-connected set that sees both $a b$ and $c d$, contradicting the maximality of $A$. Let $s$ and $s^{\prime}$ be the neighbors of $x_{i}$ in $H_{a c}$ which are closest to, respectively, $a$ and $c$ in $H_{a c}$. Let $t$ and $t^{\prime}$ be the neighbor of $x_{i}$ in $H_{b d}$ which are closest to, respectively, $d$ and $b$ in $H_{b d}$. Suppose that $s, s^{\prime}, t, t^{\prime}$ are all contained in $\{a, b, c, d\}$. Since $x_{i}$ does not see both or exactly one of $a b, c d$, we can assume w.l.o.g. that $s=a$ and $t^{\prime}=d$. Hence $\left(a, b, H_{b d}, d, x_{i}, a\right)$ is an odd hole. Therefore we can assume w.l.o.g. that $s$ is in the interior of $H_{a c}$. Suppose $t=b$. Then $s^{\prime}$ is in the interior of $H_{a c}$, otherwise $\left(b, H_{b d}, d, c, x_{i}, b\right)$ is an odd hole. Hence, by symmetry, we can assume that $t \neq b$. Let $H_{a s}$ and $H_{d t}$ be, respectively, the paths between $a$ and $s$ and $d$ and $t$ in $H \backslash b$.

If the distance between $s$ and $t$ in $H$ is even, then $F=a, H_{a s}, s, x_{i}, t, H_{d t}, d$ is an odd chordless path (since $a$ and $d$ have odd distance in $H$ ). Since $a$ and $d$ are both universal for $A$ and no node in $F \backslash\{a, d\}$ is universal for $A$, then by Lemma 6 and Remark $7 F$ has length 3, hence $F=a, s, x_{i}, d$ and there exists an odd chordless co-path $Q$ between $x_{i}$ and $s$ contained in $A \cup\left\{x_{i}, s\right\}$. If $b$ or $c$ is adjacent to neither $x_{i}$ nor $s$, then $C=(b, q, Q, r, b)$ or $C^{\prime}=(c, q, Q, r, c)$ is an odd anti-hole, a contradiction. Hence both $b$ and $c$ are adjacent to $x_{i}$ or $s$. Since $b$ cannot be adjacent to $s$, then $b$ is adjacent to $x_{i} . x_{i}$ is adjacent to $s$, $b$ and $d$ in $H$, hence $\left(H, x_{i}\right)$ must be a triangle-free wheel, therefore $x_{i}$ is not adjacent to $c$, thus $c$ is adjacent to $s$. But then $V(Q) \cup\{a, b, c, d\}$ induces the complement of a long prism, namely a $3 P C\left(a c x_{i}, d b s\right)$, a contradiction.

Therefore $s$ and $t$ must have odd distance in $H$. One can readily verify that this implies that $\left(H, x_{i}\right)$ has to be a line wheel. Furthermore, given $s^{\prime}$ and $t^{\prime}$ the neighbors of $x_{i}$ in $H$ adjacent to, respectively, $s$ and $t, s s^{\prime}$ must be an edge of $H_{a c}$ and $t t^{\prime}$ must be an edge of $H_{b d}$. Since $A$ contains node $v$ and $(H, v)$ is a line wheel, and since $A \cup x_{i}$ is co-connected, then let $y$ be a node in $A$ such that $(H, y)$ is a line wheel and $y$ has minimum distance from $x_{i}$ in $\bar{G}\left[A \cup x_{i}\right]$. Let $Q$ be a shortest co-path between $x_{i}$ and $y$ in $A \cup x_{i}$. If $Q$ has length one, then $\left(H, x_{i}, y\right)$ is a double line wheel, a contradiction. Hence $Q$ has length strictly greater than one. Since $x_{i}$ is not adjacent to both $b$ and $d$, we may assume, w.l.o.g., that $x_{i}$ is not adjacent to $d$. Since $s$ is adjacent to neither $b$ nor $d$, then, since every node of $Q \backslash\left\{x_{i}, y\right\}$ is universal for $A, C=\left(s, y, Q, x_{i}, d, s\right)$ is an anti-hole, hence $Q$ must have odd length. If $H$ has length greater than 6 , then either $H_{a c}$ or $H_{b d}$ had length at least 4 , hence there exists a node $w$ in $H \backslash\left\{a, b, c, d, s, s^{\prime}, t, t^{\prime}\right\}$. Since such a node is universal for $Q \backslash\left\{x_{i}, y\right\}$ and $Q$ has odd length strictly greater than one, then $C=\left(w, x_{i}, Q, y, w\right)$ is an odd anti-hole, a contradiction. Hence $H$ has length 6 and $H=(a, b, t, d, c, s)$, where $b=t^{\prime}$ and $c=s^{\prime}$. But then $V(Q) \cup\{a, d, s, t\}$ induces the complement of a long prism, namely a $3 P C\left(\right.$ sty, dax $\left._{i}\right)$.

Case 2: $x_{i}$ has only two neighbors in $H$ and they are adjacent.

Then, w.l.o.g., $i=1$ and $x_{1}$ is adjacent to both $c$ and $d$. Then $n>1$. Let $x_{j}$ be the node of lowest index in $P \backslash x_{1}$ to have a neighbor in $H$. Node $x_{j}$ cannot be adjacent to $c$ by the definition of $P$. Suppose $x_{j}$ is not adjacent to $d$. If $x_{j}$ has a unique neighbor $t$ in $H$, then there is a $3 P C\left(c d x_{1}, t\right)$, if $x_{j}$ has two nonadjacent neighbors in $H$, then 
there is a $3 P C\left(c d x_{1}, x_{j}\right)$, hence $x_{j}$ has exactly two neighbors $t$ and $t^{\prime}$ in $H$ and they are adjacent, but then there is a long prism, namely a $3 P C\left(c d x_{i}, t t^{\prime} x_{j}\right)$, a contradiction. So $x_{j}$ is adjacent to $d$. Suppose that $a$ has a neighbor in $P$. Let $x_{k}$ be the node of lowest index adjacent to $a$. By Claim $1, k=n$. Since $x_{n}$ has a neighbor in $H_{b d}$, then by Case $1, x_{n}$ is adjacent to $a$ and $b$ but no other node of $H$. Hence the nodes of $H$ together with a subset of the nodes of $P_{x_{j} x_{n}}$ induce a $3 P C\left(a b x_{n}, d\right)$. Therefore $a$ has no neighbors in $P$. Let $F$ be the shortest path between $c$ and $b$ in $P \cup H \backslash\{a, d\}$. Then $F$ is an odd path, otherwise $H^{\prime}=\left(c, F, b, a, H_{a c}, c\right)$ would be an odd hole. Since $b$ and $c$ are universal for $A$ and no node in $F$ is universal for $A$, then by Lemma 6 and Remark $7 F$ has length 3 , hence $F=c, x_{1}, x_{2}, b$ and there exists an odd chordless co-path $Q$ between $x_{1}$ and $x_{2}$ contained in $A \cup\left\{x_{1}, x_{2}\right\}$. But then $C=\left(a, x_{1}, Q, x_{2}, a\right)$ is an odd anti-hole, a contradiction. This concludes the proof of Claim 2.

By Claim 2, $n>1, x_{1}$ has no neighbors in $H_{b d}$ and $x_{n}$ has no neighbors in $H_{a c}$. By Claim 1 and 2, we can assume that $a$ has no neighbors in $P \backslash x_{1}$. Suppose that $\left(H, x_{1}\right)$ is not a cap. Let $t$ be the neighbor of $x_{1}$ closest to $a$ in $H_{a c}$ and $t^{\prime}$ be the neighbor of $x_{1}$ closest to $c$ in $H_{a c}$ (possibly $t=t^{\prime}$ ). Let $H_{a t}$ and $H_{c t^{\prime}}$ be, respectively, the paths between $a$ and $t$ and $c$ and $t^{\prime}$ in $H_{a c}$. Since $\left(H, x_{1}\right)$ is not a cap, then $H_{a t}$ and $H_{c t^{\prime}}$ have the same parity. Let $s$ be the neighbor of $x_{n}$ closest to $b$ in $H_{b d}$, and $H_{b s}$ the path between $s$ and $b$ in $H_{b d}$. Since $a$ has no neighbors in $P \backslash x_{1}$, then $H^{\prime}=\left(a, H_{a t}, t, x_{1}, P, x_{n}, s, H_{b s}, b, a\right)$ is a hole, hence $H^{\prime}$ has even length. Since $H_{a t}$ and $H_{c t^{\prime}}$ have the same parity, then $F=c, H_{c t^{\prime}}, t^{\prime}, x_{1}, P, x_{n}, s, H_{b s}, b$ is an odd chordless path. Since $b$ and $c$ are universal for $A$ and no intermediate node in $F$ is universal for $A$, then by Lemma 6 and Remark $7 F$ has length 3 , hence $F=c, x_{1}, x_{2}, b$, and there exists an odd chordless co-path $Q$ between $x_{1}$ and $x_{2}$ in $A \cup\left\{x_{1}, x_{2}\right\}$. If $a$ is not adjacent to $x_{1}$, then $C=\left(a, x_{1}, Q, x_{2}, a\right)$ is an odd anti-hole. Analogously, if $d$ is not adjacent to $x_{2}$, then $C^{\prime}=\left(d, x_{1}, Q, x_{2}, d\right)$ is an odd anti-hole. Hence $a$ is adjacent to $x_{1}$ and $d$ is adjacent to $x_{2}$. But then $V(Q) \cup\{a, b, c, d\}$ induces the complement of a long prism, namely a $3 P C\left(x_{1} b d, x_{2} a c\right)$, a contradiction.

Therefore $\left(H, x_{1}\right)$ is a cap and, by symmetry, $\left(H, x_{n}\right)$ is a cap. Let $t$ and $t^{\prime}$ be the attachments of $x_{1}$ in $H$ and $s$ and $s^{\prime}$ be the attachments of $x_{n}$ in $H$. If $a$ has a neighbor in $P \backslash x_{1}$, then by Claim 1 no node of $P \backslash\left\{x_{1}, x_{n}\right\}$ is adjacent to $d$, hence there exists a $3 P C\left(x_{n} s s^{\prime}, a\right)$, a contradiction. So $a$ has no neighbors in $P \backslash x_{1}$ and, by symmetry, $d$ has no neighbors in $P \backslash x_{n}$, but then $V(H) \cup V(P)$ induces a long prism, namely a $3 P C\left(x_{1} t t^{\prime}, x_{n} s s^{\prime}\right)$, a contradiction.

\subsection{Big universal wheels}

Lemma 9 implies that Theorem 5 holds whenever $G$ or $\bar{G}$ contains a line wheel. Hence, from now on, we will assume that $G$ and $\bar{G}$ do not contain any line wheel.

Lemma 10 If $G$ contains a big universal wheel, then $G$ has a T-cutset. 
Proof: Assume $G$ contains a universal wheel $(H, x)$ and let $A$ be a maximal co-connected set of $G \backslash V(H)$ such that every node in $A$ is universal for $V(H)$. Consider a bicoloring of the nodes of $H$ obtained by coloring the nodes of $H$ red and blue in such a way that two nodes have the same color if and only if they have even distance in $H$. Let $y$ be a node in $G \backslash(V(H) \cup A)$ that is not universal for $A$ such that $y$ has two nonadjacent neighbors in $H$. We will show that $(H, y)$ is a triangle-free wheel and $y$ is universal for either the red or the blue nodes of $H$. Let $u$ be a node in $A$ that is not adjacent to $y$. By the maximality of $A, y$ is not universal for $V(H)$, hence $y$ has two consecutive nonadjacent neighbors $s$ and $t$ in $H$. Let $H_{s t}$ be a path between $s$ and $t$ in $H$ containing no neighbors of $y$. Then $s$ and $t$ have distance 2 in $H_{s t}$, otherwise $H^{\prime}=\left(y, s, H_{s t}, t, y\right)$ is a big hole and $\left(H^{\prime}, u\right)$ is a proper wheel (since $u$ is adjacent to every node but $y$ in $H^{\prime}$ ). Hence $(H, y)$ is not a twin wheel, so $H \cup y$ is a triangle-free graph in which every pair of consecutive neighbors of $y$ in $H$ has distance two in $H$. Hence $y$ is either universal for the red or for the blue nodes of $H$. So we can partition the nodes in $G \backslash(V(H) \cup A)$ that have two nonadjacent neighbors in $H$ and that are not universal for $A$ into sets $\Delta_{R}$ and $\Delta_{B}$, where every node in $\Delta_{R}$ (resp. $\Delta_{B}$ ) is universal for the red (resp. blue) nodes of $H$ and has no blue (resp. red) neighbor in $H$. Next, we will show that either $\Delta_{R}$ or $\Delta_{B}$ is empty. Assume not and let $r$ and $b$ be two nodes in $\Delta_{R}$ and $\Delta_{B}$ respectively. Let st and $s^{\prime} t^{\prime}$ be two nonadjacent edges of $H$ where $s, s^{\prime}$ are red and $t, t^{\prime}$ are blue. If $r$ and $b$ are not adjacent, then $H^{\prime}=\left(r, s, t, b, t^{\prime}, s^{\prime}, r\right)$ is a 6-hole and $\left(H^{\prime}, u\right)$ is a proper wheel or a line wheel for every node $u$ in $A$ that is not adjacent to $r$ or $b$. So $r$ and $b$ are adjacent and, since neither of them is universal for $A$, then $\bar{G}[A \cup\{r, b\}]$ contains a chordless path $Q$ between $r$ and $b . \bar{G}\left[V(Q) \cup\left\{s, s^{\prime}, t, t^{\prime}\right\}\right]$ is a long prism, namely a $3 P C\left(r t t^{\prime}, b s^{\prime} s\right)$, a contradiction.

Therefore we may assume, w.l.o.g., that every node in $G \backslash(V(H) \cup A)$ that has two nonadjacent neighbors in $H$ and that is not universal for $A$ is universal for the blue nodes of $H$ and has no red neighbor in $H$. Let $a$ be a red node of $H$ and let $b_{1}$ and $b_{2}$ be its neighbors in $H$. Let $B$ be the set of all nodes in $G \backslash(A \cup V(H)) \cup\left\{b_{1}, b_{2}\right\}$ that are universal for $A$. If $a$ and $V(H) \backslash\left\{a, b_{1}, b_{2}\right\}$ lie in distinct connected components of $G \backslash(A \cup B)$, let $C$ be the connected component of $G \backslash(A \cup B)$ containing $a$ and $D=V(G) \backslash(A \cup B \cup C)$. Then $(A, B, C, D)$ is a skew partition and, given a node $t$ in $V(H) \backslash\left\{a, b_{1}, b_{2}\right\}$, then $t \in D$ and both $a$ and $t$ are universal for $A$, hence $A \cup B$ is a T-cutset. Hence we may assume that there exists a chordless path $P=x_{1}, \ldots, x_{n}$ in $G \backslash(A \cup B \cup V(H))$ such that $x_{1}$ is adjacent to $a, x_{n}$ has a neighbor in $V(H) \backslash\left\{a, b_{1}, b_{2}\right\}$ and no intermediate node has a neighbor in $V(H) \backslash\left\{b_{1}, b_{2}\right\}$. Note that $x_{1}$ does not have two nonadjacent neighbors in $H$, hence $n>1$. Also, no node in $P \backslash x_{n}$ has two nonadjacent neighbors in $H$. Note that $b_{1}$ and $b_{2}$ cannot both have neighbors in $P \backslash x_{n}$, otherwise let $x_{i}$ and $x_{j}$ be neighbors of $b_{1}$ and $b_{2}$, respectively, in $P \backslash x_{n}$ such that $x_{i}$ and $x_{j}$ are closest possible in $P \backslash x_{n}$. Then $H^{\prime}=H \backslash a \cup V\left(P_{x_{i} x_{j}}\right)$ is a hole and, for any node $u \in A$ that is not universal for $P_{x_{i} x_{j}}$, $\left(H^{\prime}, u\right)$ is a proper wheel. Thus we may assume that $b_{1}$ has no neighbors in $P \backslash x_{n}$.

If $x_{n}$ has only blue neighbors in $H$, then let $t$ be the closest neighbor of $x_{n}$ to $b_{1}$ and $H_{b_{1} t}$ be the chordless path between $b_{1}$ and $t$ in $H \backslash a . H^{\prime}=\left(a, x_{1}, P, x_{n}, t, H_{b_{1} t}, b_{1}, a\right)$ is a 
hole of even length, hence, since $t$ and $a$ have odd distance, $P$ is an odd path. Let $s$ be a neighbor of $x_{n}$ distinct from $b_{1}$ and $b_{2}$. Then $F=a, x_{1}, P, x_{n}, s$ is an odd chordless path and $F \backslash\{a, s\}$ does not have any node universal for $A$. By Lemma 6 and Remark $7, F$ has length 3 and $A \cup\left\{x_{1}, x_{2}\right\}$ contains an odd chordless co-path $Q$ between $x_{1}$ and $x_{2}$. Let $w$ be a red node distinct from $a$. Then $C=\left(a, x_{1}, Q, x_{2}, a\right)$ is an odd anti-hole, a contradiction.

So $x_{n}$ has a red neighbor in $H$, therefore $x_{n}$ does not have two nonadjacent neighbors in $H$. Let $t$ be the unique red neighbor of $x_{n}$ in $H$. Since $|H| \geq 6, t$ is not adjacent to $b_{1}$ or $b_{2}$, say $b_{i}$ for $i=1$ or 2 . Let $H_{b_{i} t}$ be the path between $b_{i}$ and $t$ in $H \backslash a$. Since $t$ and $b_{i}$ have distinct colors, $H_{b_{i} t}$ has odd length, so $\left|H_{b_{i} t}\right| \geq 3$. If $x_{n}$ has no neighbors in $H_{b_{i} t}$, then let $u$ be a node of $A$ that is not adjacent $x_{n}$. If $b_{i}$ has no neighbors in $P$, then $H^{\prime}=\left(t, H_{b_{i} t}, b_{i}, a, x_{1}, P, x_{n}, t\right)$ is a big hole and $\left(H^{\prime}, u\right)$ is a proper wheel. Otherwise let $x_{j}$ be the node of highest index in $P$ adjacent to $b_{i}$. Then $H^{\prime \prime}=\left(t, H_{b_{i} t}, b_{i}, x_{j}, P_{x_{j} x_{n}}, x_{n}, t\right)$ is a big hole and $\left(H^{\prime \prime}, u\right)$ is a proper wheel. So $x_{n}$ has exactly two neighbors $s$ and $t$ in $H$, $s$ and $t$ are adjacent and $s$ is in $H_{b_{i}}$, so $s \neq b_{1}, b_{2}$. So $s$ and $t$ have no neighbor in $P \backslash x_{n}$. Let $x_{j}$ be a node of highest index with a neighbor in $\left\{a, b_{2}\right\}$. We already observed that $x_{j}$ cannot have two nonadjacent neighbors in $H$. If $x_{j}$ has a unique neighbor $v$ in $\left\{a, b_{2}\right\}$, then there is a $3 P C\left(x_{n} s t, v\right)$. So $j=1$ and $x_{1}$ is adjacent to $b_{2}$ and there is a long prism $3 P C\left(x_{1} a b_{2}, x_{n} t s\right)$, a contradiction.

\subsection{Caps}

By Lemmas 9 and 10, we may assume $G$ and $\bar{G}$ do not contain any long prism or any big wheel except twin wheels and triangle-free wheels.

Lemma 11 If $G$ contains a big cap, then $G$ has a good skew-partition.

Before proving Lemma 11, we shall prove the following three lemmas.

Lemma 12 Let $\Gamma$ be a Berge graph. If $\Gamma$ and $\bar{\Gamma}$ do not contain any big wheel $(H, x)$ where $x$ has more than $|H| / 2$ neighbors in $H$, then $\Gamma$ does not contain both a big hole and a big anti-hole.

Proof: Assume, by contradiction, that $\Gamma$ contains a hole $H$ and an anti-hole $A$, where $n=|H| \geq 6$ and $m=|A| \geq 6$.

Assume first that $V(H) \cap V(A) \neq \emptyset$. It is immediate to verify that $|V(H) \cap V(A)| \leq 4$ and $V(H) \cap V(A)$ induces a chordless path or the complement of a chordless path. W.l.o.g., assume $P=\Gamma[V(H) \cap V(A)]$ is a chordless path, and let $k=|V(H) \cap V(A)|$.

By assumption, every node in $V(A) \backslash V(H)$ has at most $n / 2$ neighbors in $H$, hence there are at most $(m-k) n / 2$ edges between $V(H)$ and $V(A) \backslash V(H)$. Since $P$ has $k$ nodes and $k-1$ edges, and every node in $A$ has exactly $m-3$ neighbors in $A$, then between $V(A) \cap V(H)$ and $V(A) \backslash V(H)$ there are exactly $k(m-3)-2(k-1)=k m-5 k+2$ edges, hence there are at most $(m-k) n / 2-(k m-5 k+2)$ edges between $V(A) \backslash V(H)$ and $V(H) \backslash V(A)$. 
Analogously, every node in $V(H) \backslash V(A)$ has at least $m / 2$ neighbors in $V(A)$, hence there are at least $(n-k) m / 2$ edges between $V(H) \backslash V(A)$ and $V(A)$. Also, there are exactly 2 edges between $V(A) \cap V(H)$ and $V(H) \backslash V(A)$, hence there are at least $(n-k) m / 2-2$ edges between $V(A) \backslash V(H)$ and $V(H) \backslash V(A)$. Therefore

$$
\frac{(n-k) m}{2}-2 \leq \frac{(m-k) n}{2}-k m+5 k-2
$$

implying $n+m \leq 10$, that is a contradiction since $n, m \geq 6$.

Hence we may assume that $A$ and $H$ are node disjoint. Every node in $A$ has at most $n / 2$ neighbors in $H$, hence there are at most $m n / 2$ edges between $V(A)$ and $V(H)$. Every node in $H$ has at least $m / 2$ neighbors in $A$, hence there are at least $m n / 2$ edges between $V(A)$ and $V(H)$, therefore there are exactly $m n / 2$ edges between $V(A)$ and $V(H)$, every node in $V(A)$ has exactly $n / 2$ neighbors in $V(H)$ and every node in $V(H)$ has exactly $m / 2$ neighbors in $V(A)$. Let $x$ be a node of $A$. If $(H, x)$ is not a triangle-free wheel, then $H \cup x$ contains a hole $H^{\prime}$ of length at least 6 containing $x$, but $H^{\prime}$ and $A$ have one node in common and we already showed that this is not possible. Hence, for every $x \in V(A)$, $(H, x)$ is a triangle-free wheel. Let $X$ and $Y$ be the two stable sets of size $n / 2$ partitioning $V(H)$, then for every $x \in V(A)$ either $x$ is universal for $X$ and has no neighbors in $Y$, or vice-versa. Since every node in $H$ has neighbors in $A$ and $A$ is co-connected, there exist two non adjacent nodes $x$ and $y$ in $A$ such that $x$ is universal for $X$ and has no neighbors in $Y$ while $y$ is universal for $Y$ and has no neighbors in $X$. Let $x^{\prime} y^{\prime}$ and $x^{\prime \prime} y^{\prime \prime}$ be two nonadjacent edges of $H$ with $x^{\prime}, x^{\prime \prime} \in X$ and $y^{\prime}, y^{\prime \prime} \in Y$, then $H^{\prime}=\left(x, x^{\prime}, y^{\prime}, y, y^{\prime \prime}, x^{\prime \prime}, x\right)$ is a 6 -hole and $H^{\prime}$ and $A$ have two nodes in common, a contradiction.

Note that, since $G$ and $\bar{G}$ do not contain any big wheel except twin wheels and triangle-free wheels, then neither $G$ nor $\bar{G}$ contains a big wheel $(H, x)$ where $x$ has more than $|H| / 2$ neighbors in $H$. Hence, by Lemma 12, we may assume, w.l.o.g., that $G$ contains no big anti-hole.

Lemma 13 Assume $G$ contains a cap $(H, x)$ and let $a, b$ denote the attachments of $x$ in $H$. Let $P=x_{1}, \ldots, x_{n}$ be a direct connection between $x$ and $V(H) \backslash\{a, b\}$ contained in $G \backslash H$ such that no node of $P$ is adjacent to $a$. Then $x_{1}$ is adjacent to $b$ and no other node of $P$ is adjacent to $b$.

Proof: Assume first that $b$ has no neighbors in $P$. If $x_{n}$ has a unique neighbor $t$ in $H$, then there is a $3 P C(a b x, t)$. If $x_{n}$ has two nonadjacent neighbors in $H$, then there is a $3 P C\left(a b x, x_{n}\right)$. Hence $x_{n}$ has exactly two neighbors $t$ and $t^{\prime}$ in $H$ and they are adjacent, but then either $G$ contains a long prism $3 P C\left(a b x, t t^{\prime} x_{n}\right)$, or $|H|=4, n=1$ and $V(H) \cup\left\{x, x_{1}\right\}$ induces an anti-hole of length 6 . Therefore $b$ has a neighbor in $P$. If $n=1$ we are done, hence we may assume $n \geq 2$. Let $t$ be the closest neighbor of $x_{n}$ to $a$ in $H$ and $H_{t a}$ be the path between $a$ and $t$ in $H \backslash b$, let $H^{\prime}=\left(x, x_{1}, P, x_{n}, t, H_{t a}, a, x\right)$. Since $n \geq 2, H^{\prime}$ has length at least 6 and $b$ is adjacent to $a, x$ and some other node of $P$, so $\left(H^{\prime}, b\right)$ is a big wheel that is not triangle-free, hence it must be a twin wheel, therefore $b$ must be adjacent to $x_{1}$ and no other node of $P$. 
Lemma 14 Assume $G$ contains a connected set $S$, a chordless co-path $Q=y_{1}, \ldots, y_{n}$ disjoint from $S$ such that $y_{1}$ and $y_{n}$ have no neighbors in $S$ and for every $i, 2 \leq i \leq n-1$, $y_{i}$ has a neighbor in $S$. Then $n \leq 4$.

Proof: Assume, by contradiction, that $n \geq 5$. Since $y_{2}$ and $y_{3}$ have at least a neighbor in $S$ and $S$ is connected, there exists a chordless path $Q=y_{2}, q_{1}, \ldots, q_{m}, y_{3}$ between $y_{2}$ and $y_{3}$ whose interior is contained in $S$. $Q$ has even length, otherwise $y_{n}, y_{2}, Q, y_{3}$ is an odd hole, and $Q$ has length at least 4 , otherwise $Q=y_{2}, q_{1}, y_{3}$ and, given $h$ the smallest index such that $y_{h}$ is nonadjacent to $q_{1}$ ( $h$ is well defined since $y n$ has no neighbors in $S)$, then $y_{1}, P_{y_{1} y_{h}}, y_{h}, q_{1}, y_{1}$ is an antihole of length at least 6 , a contradiction. Hence $Q^{\prime}=y_{2}, Q, y_{3}, y_{1}$ is an odd path of length at least 5 and $X=V(P) \backslash\left\{y_{1}, y_{2}, y_{3}\right\}$ is a co-connected set universal for $y_{1}$ and $y_{2}$. By Lemma 6 and Remark 7, the interior of $Q^{\prime}$ must contain a node universal for $X$, a contradiction since $y_{n}$ has no neighbors in the interior of $Q$ and $y_{3}$ is not adjacent to $y_{4}$.

Proof of Lemma 11:

Consider a big cap $(H, v)$ and let $a, b$ be the attachments of $x$ in $H$. Let $a_{0}=a, P^{0}$ be the path induced by $V(H) \backslash b, A_{0}=\left\{a_{0}\right\}$ and $S_{0}=V\left(P^{0}\right) \backslash a_{0}$.

Let $P^{0}, \ldots, P^{k}$ be a sequence of chordless paths in $G$, where $P^{i}=x_{1}^{i}, \ldots, x_{l_{i}}^{i}$. For every $i, 1 \leq i \leq k$, let $a_{i}=x_{1}^{i}, A_{i}=A_{i-1} \cup a_{i}$ and $S_{i}=S_{i-1} \cup V\left(P^{i}\right) \backslash a_{i}$. Assume that the sequence $P^{0}, \ldots, P^{k}$ satisfies the following properties:

1. $P^{i}$ is a direct connection between $x$ and $S_{i-1}$ contained in $G \backslash\left(A_{i-1} \cup\{b\}\right)$ such that no node in $P^{i}$ is universal for $A_{i-1}$,

2. For every $i, 0 \leq i \leq k, a_{i}$ is adjacent to $b$.

We will prove that, if $P=x_{1}, \ldots, x_{n}$ is a direct connection between $x$ and $S_{k}$ contained in $G \backslash\left(A_{k} \cup b\right)$ such that no node in $P$ is universal for $A_{k}$, then $x_{1}$ is adjacent to $b$.

Note that this implies Lemma 11: obviously, $P^{0}$ is a sequence satisfying properties 1 and 2 above, hence we can consider a sequence $P^{0}, \ldots, P^{k}(k \geq 0)$, that is the longest possible. Let $A=A_{k}$ and $B$ be the set of all nodes in $V \backslash x$ that are universal for $A$. If $A \cup B$ is not a skew cutset that separates $x$ from $S_{k}$, then there exists a direct connection $P=x_{1}, \ldots, x_{n}$ between $x$ and $S_{k}$ contained in $G \backslash\left(A_{k} \cup b\right)$ such that no node in $P$ is universal for $A_{k}$. Since $x_{1}$ is adjacent to $b$, we can choose $P^{k+1}=P$. Now $P^{0}, \ldots, P^{k+1}$ is a sequence satisfying properties 1 and 2, contradicting the maximality of $k$. Hence $A \cup B$ is a skew cutset that separates $x$ from $S_{k}$. Let $C$ be the connected component of $G \backslash(A \cup B)$ containing $x$ and $D=V(G) \backslash(A \cup B \cup C)$. Then $(A, B, C, D)$ is a skew partition and $x$ is universal for $A$, hence it is a good skew partition.

Observe that, by construction, for every $0 \leq i \leq k, A_{i}$ is a co-connected set and $S_{i}$ is connected. Moreover, $x$ has no neighbors in $S_{k}$ and every node in $A_{i}$ has a neighbor in $S_{i}$. 
Claim 1: For every $j, 1 \leq j \leq k$, and for every node $y \in S_{j}$, if $y$ is universal for $A_{j-1}$, then $y$ is the only neighbor of $a_{0}$ in $H \backslash b$.

Proof of Claim 1: By construction, for every $i$ such that $1 \leq i \leq j$, no node in $P^{i}$ is universal for $A_{j-1}$, hence $y$ must be the node in $P^{0}$ adjacent to $a_{0}$.

Claim 2: For every $i, 0 \leq i \leq k, b$ does not see any edge of $P^{i}$.

Proof of Claim 2: The statement is trivial for $i=0$ and it follows immediately by Lemma 13 for $i=1$. Hence we may assume $i \geq 2$. Assume, by contradiction, that $b$ sees an edge $x_{j}^{i} x_{j+1}^{i}$ of $P^{i}$. We will show that every node of $A_{i-1}$ is adjacent to $x_{j}^{i}$ or $x_{j+1}^{i}$. Assume not, then there exists $a_{h} \in A_{i-1}$ such that $a_{h}$ is not adjacent to $x_{j}^{i}$ and $x_{j+1}^{i}$. Let $y$ be the neighbor of $a_{h}$ in the chordless path $x, x_{1}^{i}, P_{x_{1}^{i} x_{j-1}^{i}}^{i}, x_{j-1}^{i}$ closest to $x_{j}^{i}$ in $P^{i}$ ( $y$ is well defined since every node in $A_{k}$ is adjacent to $x$ ) and let $F$ be the path from $y$ to $x_{j}^{i}$ in $x, x_{1}^{i}, P_{x_{1}^{i} x_{j}^{i}}^{i}, x_{j}^{i}$. Let $F^{\prime}$ be a chordless path between $a_{h}$ and $x_{j+1}^{i}$ in the graph induced by $S_{i-1} \cup\left(a_{h} \cup V\left(P_{x_{j+1}^{i} x_{l_{i}}^{i}}^{i}\right)\right)$. By construction, no node in $P^{i}$ except $x_{l_{i}}^{i}$ has a neighbor in $S_{i-1}$, hence $C=\left(a_{h}, y, F, x_{j}^{i}, x_{j+1}^{i}, F^{\prime}, a_{h}\right)$ is a big hole and $b$ is adjacent to $a_{h}, x_{j}^{i}$ and $x_{j+1}^{i}$ in $C$, hence $(C, b)$ is a big wheel that is neither a triangle-free nor a twin wheel, a contradiction.

Hence every node in $A_{i-1}$ is adjacent to $x_{j}^{i}$ or $x_{j+1}^{i}$ but no node in $P^{i}$ is universal for $A_{i-1}$, so there exists a chordless co-path $Q=y_{1}, \ldots, y_{m}$ in $G\left[A_{i-1}\right]$ such that $y_{1}$ is adjacent to $x_{j}^{i}$ but not $x_{j+1}^{i}, y_{m}$ is adjacent to $x_{j+1}^{i}$ but not $x_{j}^{i}$ and all the intermediate neighbors of $Q$ are adjacent to both $x_{j}^{i}$ and $x_{j+1}^{i}$. If $j>1$, then $x_{j}^{i}$ and $x_{j+1}^{i}$ are not adjacent to $x$, hence $\left(x, x_{j+1}^{i}, y_{1}, Q, y_{m}, x_{j}^{i}, x\right)$ is a big anti-hole. Therefore $j=1$, and $Q^{\prime}=x, x_{2}^{i}, y_{1}, Q, y_{m}, x_{1}^{i}$ is a co-path of length at least 4 . Let $S=\left(S_{i-1} \cup V\left(P^{i}\right)\right) \backslash\left\{x_{1}^{i}, x_{2}^{i}\right\} . S$ is connected and neither $x$ nor $x_{1}^{i}$ have neighbors in $S$, while, by construction, every intermediate node of $Q^{\prime}$ has a neighbor in $S$. Now $Q^{\prime}$ and $S$ contradict Lemma 14. This completes the proof of Claim 2.

We will prove Lemma 11 by induction on $k$. If $k=0$, then we are done by Lemma 13 . Let us now assume, by induction, that the statement is satisfied for every big cap $(H, x)$, and for every sequence $P_{0}, \ldots, P_{j}$ satisfying properties 1 and 2 , whenever $j \leq k-1$. Note that $P$ must contain a node that is universal for $A_{k-1}$, otherwise $\left(V(P) \cup V\left(P^{k}\right)\right) \backslash a_{k}$ contains a direct connection $P^{\prime}$ from $x$ to $S_{k-1}$ and no node in $P^{\prime}$ is universal for $A_{k-1}$ so, by induction, the first node of $P^{\prime}$, which is $x_{1}$, is adjacent to $b$ and we are done. Let us assume, by contradiction, that $x_{1}$ is not adjacent to $b$.

Claim 3: No node of $P$ is adjacent to $a_{k}$.

Proof of Claim 3: Assume, by contradiction, that $a_{k}$ has a neighbor in $P$. Then, since by the argument above $P$ contains a node universal for $A_{k-1}$, every node in $A_{k}$ has a neighbor in $P$. For every $0 \leq i \leq k$, let $h(i)$ be the minimum index such that $a_{i}$ is adjacent to $x_{h(i)}$ and let $h=\max _{0 \leq i \leq k} h(i)$. Since no node of $P$ is universal for $A_{k}, h \geq 2$. If $h=2$, then every node in $A_{k}$ is adjacent to $x_{1}$ or $x_{2}$ but neither $x_{1}$ or $x_{2}$ are universal for $A_{k}$, hence $A_{k}$ contains a chordless co-path $Q=y_{1}, \ldots, y_{m}$ such that $y_{1}$ is adjacent to 
$x_{1}$ but not to $x_{2}, y_{m}$ is adjacent to $x_{2}$ but not $x_{1}$ and every intermediate node of $Q$ is adjacent to both $x_{1}$ and $x_{2}$. Therefore $Q^{\prime}=x, x_{2}, y_{1}, Q, y_{m}, x_{1}$ is a co-path of length at least 4. Let $S=S_{k} \cup\left(V(P) \backslash\left\{x_{1}, x_{2}\right\}\right)$. $S$ is a connected set and neither $x_{1}$ nor $x$ has a neighbor in $S$, while every intermediate node of $Q^{\prime}$ has a neighbor in $S$. Therefore $Q^{\prime}$ and $S$ contradict Lemma 14. Hence we can assume $h \geq 3$. Let $a_{j} \in A_{k}$ be such that $h(j)=h$. Then $C=\left(a_{j}, x, x_{1}, P_{x_{1} x_{h}}, x_{h}, a_{j}\right)$ is a big hole. Since $b$ is adjacent to both $x$ and $a_{j}$, then $(C, b)$ is either a cap or a twin wheel. If $(C, b)$ is a cap, let $F$ be a shortest path between $x_{h}$ and $b$ in $S_{k} \cup V\left(P_{x_{h} x_{n}}\right) \cup b$, then $C^{\prime}=\left(x, x_{1}, P_{x_{1} x_{h}}, x_{h}, F, b, x\right)$ is a hole and $a_{j}$ is adjacent to $x, b$ and $x_{h}$ in $C^{\prime}$, therefore $\left(C^{\prime}, a_{h}\right)$ is a big wheel that is neither a triangle-free wheel nor a twin wheel. Hence $(C, b)$ must be a twin wheel so $b$ is adjacent either to $x_{1}$ or to $x_{h}$. In the former case we are done. Now assume that $b$ is adjacent to $x_{h} . C^{\prime}=\left(x, x_{1}, P_{x_{1} x_{h}}, x_{h}, b, x\right)$ is a big hole. Since every node in $A_{k}$ has a neighbor in $P_{x_{1} x_{h}}$, then $\left(C^{\prime}, a_{i}\right)$ must be a twin wheel for every $a_{i} \in A_{k}$, hence every node in $A_{k}$ is adjacent to $x_{1}$ or $x_{h}$. Since no node in $P$ is universal for $A_{k}$ and $A_{k}$ is co-connected, there exists two nonadjacent nodes $a_{s}$ and $a_{t}$ in $A_{k}$ such that $a_{s}$ is adjacent to $x_{1}$ and not to $x_{h}$, and $a_{t}$ is adjacent to $x_{h}$ and not to $x_{1}$. $C^{\prime \prime}=\left(x, x_{1}, P_{x_{1} x_{h}}, x_{h}, a_{t}, x\right)$ is a big hole and $\left(C^{\prime \prime}, a_{s}\right)$ is a cap where $x, x_{1}$ are the attachments of $a_{s}$ in $C^{\prime \prime}$. By construction $S_{k}$ contains a direct connection $F$ form $a_{s}$ to $C^{\prime \prime} \backslash\left\{x_{1}, x\right\}$, but no node in $S_{k}$ is adjacent to $x$ or to $x_{1}$, hence $F$ contradicts Lemma 13. This completes the proof of Claim 3.

Claim 4: $b$ does not have any neighbor in $P$.

Proof of Claim 4: Assume by contradiction that $x_{j}$, for some $1 \leq j \leq n$, is adjacent to b. Let $F$ be a chordless path between $a_{k}$ and $x_{1}$ in $S_{k} \cup V(P)$. Since $a_{k}$ has no neighbor in $P$, then $P$ is a subpath of $F$ and $C=\left(x, x_{1}, F, a_{k}, x\right)$ is a hole, $b$ is adjacent to $x, a_{k}$ and $x_{j}$ in $P$ but $x_{j}$ is not adjacent to $x$ (otherwise $j=1$ and $b$ is adjacent to $x_{1}$ ) and $x_{j}$ is not adjacent to $a_{k}$ (because, by Claim $3, a_{k}$ has no neighbors in $P$ ), hence $(C, b)$ is a big wheel that is neither a twin wheel nor a triangle-free wheel, a contradiction.

Claim 5: $S_{k} \cup V(P)$ contains a chordless path $F=y_{1}, \ldots, y_{m+1}$ between $x_{1}$ and $b$ such that $a_{k}$ is adjacent to $y_{m}$ and no other node in $F$ and $y_{1}$ is universal for $A_{k-1}$.

Proof of Claim 5: Let $F=y_{1}, \ldots, y_{m+1}$ be a chordless path between $x_{1}$ and $b$ in $S_{k} \cup V(P)$, where $y_{1}=x_{1}$ and $y_{m+1}=b$. Note that, since $b$ is not adjacent to $x_{1}$, then $C=\left(x, x_{1}, F, b, x\right)$ is a hole. Since $b$ has no neighbor in $P$, then $P$ is a subpath of $F$ and $y_{m}$ is in $S_{k}$. By Claim 1, $y_{m}$ is not universal for $A_{k-1}$ since $y_{m}$ is adjacent to $b$.

Since $P$ contains a node $x_{j}$ universal for $A_{k-1}$ and $\left(C, a_{i}\right)$ is a wheel that is not triangle-free for each $a_{i} \in A_{k-1}$, every node in $A_{k-1}$ must be adjacent to $x_{1}$. If $y_{m}$ is adjacent to $a_{k}$ we are done. Otherwise $\left(C, a_{k}\right)$ is a cap where $x, b$ are the attachments of $a_{k}$ in $C$. Let $Z=z_{1}, \ldots, z_{l}$ be a direct connection form $a_{k}$ to $V(C) \backslash\{x, b\}$, contained in $S_{k} \backslash V(F)$. Since no node in $Z$ is adjacent to $x$, then by Lemma $13 z_{1}$ is adjacent to $b$ and no node in $Z \backslash z_{1}$ is adjacent to $b$. Hence, given $y$ the closest neighbor of $z_{l}$ to $x_{1}$ in $F, F^{\prime}=x_{1}, F_{x_{1} y}, z_{l}, Z, z_{1}, b$ is a chordless path between $x_{1}$ and $b$ in $S_{k} \cup V(P)$. Note that $F^{\prime}=y_{1}^{\prime}, \ldots, y_{m^{\prime}+1}^{\prime}$, where $y_{1}^{\prime}=x_{1}$ and $y_{m^{\prime}+1}^{\prime}=b$ and $a_{k}$ is adjacent to $y_{m^{\prime}}^{\prime}$ and no other node in $F^{\prime}$. Thus $F^{\prime}$ satisfies the statement of Claim 5. 
Let $j, 0 \leq j \leq k$, be the index such that $y_{m} \in V\left(P^{j}\right)$. Note that, since $y_{m}$ and $b$ are adjacent to $a_{k}$, then, by Claim $2, y_{m} \neq x_{2}^{k}$, hence $j<k$. This implies that $P^{k}$ consist of only one node, namely $a_{k}$.

Claim 6: $a_{k}$ is universal for $A_{k-2}$ and $a_{k}$ is not adjacent to $a_{k-1}$.

Proof of Claim 6: If $a_{k}$ is universal for $A_{k-2}$, then by construction $a_{k}$ is not adjacent to $a_{k-1}$. Assume, by contradiction, that $a_{k}$ is not universal for $A_{k-2}$. Then $\left(V\left(P^{k}\right) \cup\right.$ $\left.V\left(P^{k-1}\right)\right) \backslash\left\{a_{k-1}\right\}$ contains a direct connection $\tilde{P}^{k-1}=\tilde{x}_{1}^{k-1}, \ldots, \tilde{x}_{l_{k-1}^{k}}^{k-1}$ from $x$ to $S_{k-2}$ such that no node in $\tilde{P}^{k-1}$ is universal for $A_{k-2}$ (obviously, $\tilde{P}^{k-1}$ contains $P^{k}=a_{k}$ and $\left.\tilde{x}_{1}^{k-1}=a_{k}\right)$. Let $\tilde{a}_{k-1}=\tilde{x}_{1}^{k-1}, \tilde{A}_{k-1}=A_{k-2} \cup\left\{\tilde{a}_{k-1}\right\}$ and $\tilde{S}_{k-1}=S_{k-2} \cup V\left(\tilde{P}^{k-1}\right)$. Let $\tilde{P}=\tilde{x}_{1}, \ldots, \tilde{x}_{n^{\prime}}$ be a direct connection contained in $\left(V(P) \cup V\left(P^{k-1}\right)\right) \backslash\left(\tilde{S}_{k-1} \cup\left\{a_{k-1}\right\}\right)$ from $x$ to $\tilde{S}_{k-1}$. By Claim 3 and by construction of $P^{k-1}, \tilde{P}$ does not contain any node universal for $\tilde{A}_{k-1}$. But $\tilde{x}_{1}=x_{1}, x_{1}$ is not adjacent to $b$, contradicting the inductive hypothesis. This proves Claim 6.

Let $h$ be the lowest index such that $2 \leq h \leq l_{j}$ such that $x_{h}^{j}$ is adjacent to $b$ (one such index exists since $\left.y_{m} \in V\left(P^{j}\right) \backslash a_{j}\right)$.

Claim 7: $h \geq 5$ and every node in $A_{j-1}$ has a neighbor in $P_{x_{2}^{j} x_{h}^{j}}^{j}$.

Proof of Claim 7: By Claim 2, $h \geq 3$, hence $\tilde{H}=\left(b, x_{1}^{j}, P_{x_{1}^{j} x_{h}^{j}}^{j}, x_{h}^{j}, b\right)$ is a hole. We first show that every node in $A_{j-1}$ has a neighbor in $P_{x_{2}^{j} x_{h}^{j}}^{j}$. Assume not, then there exists $q, 0 \leq q \leq j-1$, such that $a_{q}$ has no neighbor in $P_{x_{2}^{j} x_{h}^{j}}^{j}$. Let $Z$ be a shortest path between $a_{q}$ and $x_{h}^{j}$ in $S_{j-1} \cup V\left(P_{x_{h}^{j} x_{l_{j}}^{j}}^{j}\right)$. Then by construction no node in $P_{x_{2}^{j} x_{h-1}^{j}}^{j}$ has a neighbor in $Z$ and $x_{1}^{j}$ has no neighbor in $Z \backslash a_{q}$. If $a_{q}$ is not adjacent to $a_{j}$, then $C=\left(x, a_{j}, P_{x_{1}^{j} x_{h}^{j}}^{j}, x_{h}^{j}, Z, a_{q}, x\right)$ is a big hole, otherwise $C^{\prime}=\left(a_{j}, P_{x_{1}^{j} x_{h}^{j}}^{j}, x_{h}^{j}, Z, a_{q}, a_{j}\right)$ is a big hole. In both cases, either $(C, b)$ or $\left(C^{\prime}, b\right)$ is a big wheel that is neither a twin wheel nor a triangle-free wheel, a contradiction. To conclude the proof of Claim 6 , we have only to show that $h \geq 5$. Note that $h$ must be odd, otherwise $\tilde{H}$ is an odd hole. Assume then, by contradiction, that $h=3$. Then, since every node in $A_{j-1}$ is adjacent to $x_{2}^{j}$ or $x_{3}^{j}$ but no node in $P^{j}$ is universal for $A_{j-1}, A_{j-1}$ contains a chordless co-path $Q=q_{1}, \ldots, q_{s}$ such that $q_{1}$ is adjacent to $x_{2}^{j}$ but not $x_{3}^{j}, q_{s}$ is adjacent to $x_{3}^{j}$ but not $x_{2}^{j}$, and every intermediate node of $Q$ is adjacent to both $x_{2}^{j}$ and $x_{3}^{j}$. But then $\left(x, x_{3}^{j}, q_{1}, Q, q_{s}, x_{2}^{j}, x\right)$ is a big anti-hole, a contradiction. This completes the proof of Claim 7 .

Let $\tilde{H}=\left(b, x_{1}^{j}, P_{x_{1}^{j} x_{h}^{j}}^{j}, x_{h}^{j}, b\right)$. By Claim 7, $\tilde{H}$ is a big hole.

Claim 8: $j<k-1$.

Proof of Claim 8: We already observed that $j<k$. Assume, by contradiction, that $j=k-1$. Let $\tilde{P}^{0}=\tilde{H} \backslash b$ and $\tilde{a}_{0}=x_{1}^{j}$. Let $\tilde{P}^{1}=\tilde{x}_{1}^{1}, \ldots, \tilde{x}_{l}^{1}$ be a direct connection between $x$ and $V\left(\tilde{P}^{0}\right) \backslash\left\{\tilde{a}_{0}\right\}$ contained in $\left\{a_{k}\right\} \cup V\left(P_{x_{h}^{j} y_{m}}^{j}\right)$. By construction, $\tilde{a}_{0}$ has no neighbors in $\tilde{P}^{1}$. Let $\tilde{a}_{1}=\tilde{x}_{1}^{1}=a_{k}$. Therefore the sequence $\tilde{P}^{0}, \tilde{P}^{1}$ satisfies properties 1 
and 2 at the beginning of the proof. Let $\tilde{P}=\tilde{x}_{1}, \ldots, \tilde{x}_{n^{\prime}}$ be a direct connection between $x$ and $\left(V\left(\tilde{P}^{0}\right) \cup V\left(\tilde{P}^{1}\right)\right) \backslash\left\{\tilde{a}_{0}, \tilde{a}_{1}\right\}$ contained in $V(F) \cup V\left(P_{x_{h}^{j} y_{m}}^{j}\right)$, where $F$ is the path found in Claim 5. Obviously, $\tilde{x}_{1}=x_{1}$, no node in $\tilde{P}$ is universal for $\left\{\tilde{a}_{0}, \tilde{a}_{1}\right\}$ and $\tilde{x}_{1}$ is not adjacent to $b$. If $k>1$, then $\tilde{x}_{1}$ not adjacent to $b$ contradicts the inductive hypothesis on $k$. So $k=1$ and $\tilde{a}_{0}=a_{0}, \tilde{H}=H, \tilde{P}^{0}=P^{0}, \tilde{a}_{1}=a_{1}, \tilde{P}^{1}=P^{1}=a_{1}$ and $\tilde{P}=P$. Then, by Claims 3 and $4, a_{1}$ and $b$ have no neighbors in $P$, by Claim $5 S_{2} \cup V(P)$ contains a chordless path $F=y_{1}, \ldots, y_{m+1}$ between $x_{1}$ and $b$ such that $a_{1}$ is adjacent to $y_{m}$ and no other node in $F, y_{1}=x_{1}$ is adjacent to $a_{0}$ and no node other node in $F \backslash y_{1}$. Hence $y_{m}$ must be the neighbor of $b$ in $H \backslash a_{0}$, so $a_{1}$ is adjacent in $H$ to $b$ and $y_{m}$ but not to $a_{0}$. If $a_{1}$ has no further neighbors in $H$, then $\left(x, a_{0}, P^{0}, y_{m}, a_{1}, x\right)$ is an odd hole, therefore $\left(H, a_{1}\right)$ must be a twin wheel and $a_{1}$ is adjacent to the neighbor $c$ of $y_{m}$ in $H \backslash b$. Since $y_{m}$ is the only neighbor of $a_{1}$ in $F$, then $c$ is not a node of $F$, hence $x_{n}$ is adjacent to $y_{m}$. $H^{\prime}=\left(x, x_{1}, P, x_{n}, y_{m}, a_{1}, x\right)$ is a hole and $\left(H^{\prime}, a_{0}\right)$ is a cap where $x, x_{1}$ are the attachments of $a_{0}$ in $H^{\prime} . H \backslash\{a, b\}$ contains a direct connection $P^{\prime}$ from $x$ to $V\left(H^{\prime}\right) \backslash\left\{x, x_{1}\right\}$ whose first node, that is the neighbor of $a$ in $H \backslash b$, is not adjacent to $x$. By Lemma 13 the first node of $P^{\prime}$ must be adjacent to $x_{1}$, hence $n=1$ and $x_{1}$ is adjacent in $H$ to $a, y_{m}$ and the neighbor of $a$ in $H \backslash b$. Therefore $\left(H, x_{1}\right)$ is a big wheel that is neither a triangle-free wheel nor a twin wheel, a contradiction. This completes the proof of Claim 8 .

Claim 9: $j>0$.

Proof of Claim 9: Assume $j=0$, then $y_{m}$ is the neighbor of $b$ in $H \backslash a$. By Claim $8, j<k-1$, so by Claim $6 a_{k}$ is adjacent to $a_{0}$. Hence, in $H, a_{k}$ is adjacent to $a_{0}, b$ and $y_{m}$, so $\left(H, a_{k}\right)$ is a twin wheel. Let $b^{\prime}=a_{k}, H^{\prime}=H \cup b^{\prime} \backslash b$ is a big hole. $\left(H^{\prime}, x\right)$ is a cap where the attachments of $x$ in $H^{\prime}$ are $a$ and $b^{\prime}$. Note that $P^{0}=H^{\prime} \backslash b^{\prime}$ and, by Claim 6, for every $i, 0 \leq i \leq k-2, a_{i}$ is adjacent to $b^{\prime}$. Now $P^{k-1}$ is a direct connection from $x$ to $S_{k-2}$ in $G \backslash\left(A_{k-2} \cup\left\{b^{\prime}\right\}\right)$ such that no node in $P^{k-1}$ is universal for $A_{k-2}$, but $a_{k-1}=x_{1}^{k-1}$ is not adjacent to $b^{\prime}$, contradicting the inductive hypothesis. This completes the proof of Claim 9.

Assume that $(H, x), P^{0}, \ldots, P^{k}, P$ and $F$ are chosen so that $j$ is largest possible, where the sequence $P^{0}, \ldots, P^{k}$ satisfies properties 1 and $2, P$ is a direct connection between $x$ and $S_{k}$ contained in $G \backslash\left(A_{k} \cup b\right)$ such that no node in $P$ is universal for $A_{k}$ and $x_{1}$ is not adjacent to $b$, and $F$ satisfies Claim 5 .

By Claim 7, the hole $\tilde{H}$ has length at least 6 and every node in $A_{j-1}$ has a neighbor in $\tilde{H} \backslash\left\{a_{j}, b\right\}$. Let $\tilde{a}_{0}=a_{j}, \tilde{P}^{0}=\tilde{H} \backslash b, \tilde{S}_{0}=V\left(\tilde{P}^{0}\right) \backslash b$ and $\tilde{A}_{0}=\left\{a_{0}\right\}$. Since $A_{j-1}$ is co-connected, there exists a bijection $\sigma$ between $\{1, \ldots, j\}$ and $\{0, \ldots, j-1\}$ such that, if we define $\tilde{a}_{i}=a_{\sigma(i)}$ for every $i, 1 \leq i \leq j$, and, for every $1 \leq q \leq j, \tilde{A}_{q}=\left\{\tilde{a}_{i} \mid 0 \leq i \leq q\right\}$, then for every $q, 1 \leq q \leq j$, $\tilde{a}_{q}$ is not universal for $\tilde{A}_{q-1}$. Note that $\tilde{A}_{j}=A_{j}$ and every node in $\tilde{A}_{j}$ has a neighbor in $\tilde{S}_{0}$. For every $i$ such that $1 \leq i \leq j$, we define $\tilde{S}_{i}=\tilde{S}_{0}$ and $\tilde{P}^{i}=\tilde{a}^{i}$.

For every $i$ such that $j<i \leq k$, let $\tilde{a}_{i}=a_{i}, \tilde{A}_{i}=A_{i}$ and define recursively, for $i=j+1$ to $k$, the path $\tilde{P}^{i}$ and the set $\tilde{S}_{i}$ has follows: $\tilde{P}^{i}=\tilde{x}_{1}^{i}, \ldots, \tilde{x}_{l_{i}^{\prime}}^{i}$ is a direct connection between $x$ and $\tilde{S}_{i-1}$ contained in $V\left(P^{i}\right) \cup S_{i-1}$, while $\tilde{S}_{i}=\left(\tilde{S}_{i-1} \cup V\left(\tilde{P}^{i}\right)\right) \backslash\left\{\tilde{x}_{1}^{i}\right\}$. By construction, 
$\tilde{S}_{i} \subseteq S_{i}, P^{i}$ is a subpath of $\tilde{P}^{i}$ and $\tilde{x}_{1}^{i}=\tilde{a}_{i}$ is adjacent to $b$. Moreover, since $\tilde{P}^{i}$ is contained in $V\left(P^{i}\right) \cup S_{i-1}$, no node in $\tilde{P}^{i}$ is universal for $\tilde{A}_{i-1}$. Let $\tilde{P}=\tilde{x}_{1}, \ldots, \tilde{x}_{n^{\prime}}$ be a direct connection from $x$ to $\tilde{S}_{k}$ contained in $V(P) \cup S_{k}$. Since $\tilde{S}_{k} \subseteq S_{k}, P$ is a subpath of $\tilde{P}$. Therefore $\tilde{x}_{1}=x_{1}$ is not adjacent to $b$. Finally, since $\tilde{P}$ is contained in $V(P) \cup S_{k}$, no node in $\tilde{P}$ is universal for $\tilde{A}_{k}$. By Claims 3 and $4, \tilde{a}_{k}$ and $b$ have no neighbors in $\tilde{P}$ and by Claim $5 \tilde{S}_{k} \cup V(\tilde{P})$ contains a chordless path $\tilde{F}=\tilde{y}_{1}, \ldots, \tilde{y}_{m^{\prime}+1}$ between $\tilde{x}_{1}$ and $b$ such that $\tilde{a}_{k}$ is adjacent to $\tilde{y}_{m^{\prime}}$ and no other node in $\tilde{F}$ and $\tilde{y}_{1}$ is universal for $A_{k-1}$. Let $j^{\prime}, 0 \leq j^{\prime} \leq k$, be the index such that $\tilde{y}_{m^{\prime}} \in V\left(\tilde{P}^{j^{\prime}}\right)$. By Claims $6-9,1 \leq j^{\prime} \leq k-2$. On the other hand, since $\tilde{S}_{j}=\tilde{S}_{0}, j^{\prime}>j$ contradicting our choice of $(H, x), P^{0}, \ldots, P^{k}, P$ and $F$ so that $j$ is largest possible.

By Lemmas 8-11, we can assume that $G$ does not contain any big cap, any big antihole or any big wheel except twin wheels and triangle-free wheels. We say that a cap is small if it is not big.

Lemma 15 If $G$ contains a small cap, then $G$ has a T-cutset.

Proof:

Claim 1: Let $(H, x)$ be a small cap where $a, b$ denote the attachments of $x$ in $H$, and let $P=x_{1}, \ldots, x_{n}$ be a direct connection from $x$ to $V(H) \backslash\{a, b\}$ in $G \backslash(V(H) \cup\{x\})$. If $a$ has no neighbors in $P$, then $n=1$ and $x_{1}$ is adjacent to both neighbors of $a$ in $H$.

Proof of Claim 1: By Lemma $13 x_{1}$ is adjacent to $b$ and no other node in $P$ is adjacent to $b$. Let $a^{\prime}$ and $b^{\prime}$ be, respectively, the neighbors of $a$ in $H \backslash b$ and the neighbor of $b$ in $H \backslash a$. If $x_{n}$ is not adjacent to $a^{\prime}$, then $H^{\prime}=\left(x, x_{1}, P, x_{n}, b^{\prime}, a^{\prime}, a, x\right)$ is a big hole and $\left(H^{\prime}, b\right)$ is a proper wheel. So $a^{\prime}$ is adjacent to $x_{n}$. If $n=1$ we are done, hence we may assume $n>1$. If $x_{n}$ is adjacent to $b^{\prime}$, then $H^{\prime \prime}=\left(x, x_{1}, P, x_{n}, a^{\prime}, a, x\right)$ is a big hole and $\left(H^{\prime \prime}, b^{\prime}\right)$ is a big cap. So $x_{n}$ is not adjacent to $b^{\prime}, C=\left(b, x_{1}, P, x_{n}, a^{\prime}, b^{\prime}, b\right)$ is a big hole and $(C, x)$ is a big cap, a contradiction. This proves Claim 1.

Let $Q=y_{1}, \ldots, y_{m}$ be the longest chordless path in $\bar{G}$. Note that the complement of a small cap is a chordless path on 5 nodes, so, if $G$ contains a small cap, then $Q$ has at least 5 nodes (i.e. $m \geq 5$ ). Let $\left(H, y_{3}\right)$ be the cap induced by $\left\{y_{i} \mid 1 \leq i \leq 5\right\}$, where $H=\left(y_{1}, y_{5}, y_{2}, y_{4}\right)$ and $y_{1}, y_{5}$ are the attachments of $y_{3}$ in $H$. Define $A$ to be a maximal co-connected set contained in $G \backslash\left\{y_{i} \mid 2 \leq i \leq 5\right\}$ such that $y_{1} \in A$ with the property that every node in $A$ is adjacent to $y_{3}, y_{4}, y_{5}$ but not $y_{2}$. Note that, for every $y \in A, Q \backslash y_{1} \cup y$ is a chordless co-path. Otherwise, there exists $j, 6 \leq j \leq m$, such that $y_{j}$ is not adjacent to $y$. Assume $j$ is the lowest such index. Then $C=\left(y, y_{2}, Q_{x_{2} x_{j}}, y_{j}, y\right)$ is a big anti hole, a contradiction. Let $B$ be the set of all nodes in $V(G) \backslash\left\{y_{3}, y_{4}\right\}$ that are universal for $A$. If $A \cup B$ is a cutset separating $y_{3}$ and $\left\{y_{2}, y_{4}\right\}$, then let $C$ be the connected component of $G \backslash(A \cup B)$ containing $y_{3}$ and let $D=V(G) \backslash(A \cup B \cup C)$. Then $(A, B, C, D)$ is a skew-partition, $y_{3} \in C$ is universal for $A$ and $y_{4} \in D$ is universal for $A$, hence $A \cup B$ is a T-cutset.

Next we will show that $A \cup B$ is a cutset separating $y_{3}$ and $\left\{y_{2}, y_{4}\right\}$. Assume not. Then there exists a direct connection $P=x_{1}, \ldots, x_{n}$ in $G \backslash(A \cup B)$ between $y_{3}$ and $\left\{y_{2}, y_{4}\right\}$. 
If there exists a node $y \in A$ with no neighbors in $P$, then consider $H^{\prime}=H \cup y \backslash y_{1} \cdot H^{\prime}$ is a hole of length 4 and $\left(H^{\prime}, y_{3}\right)$ is a small cap. By Claim $1, n=1$ and $x_{1}$ is adjacent to $y_{4}$ and $y_{5}$. If $x_{1}$ is adjacent to $y_{2}$, then $x_{1}, y, y_{2}, Q_{y_{2} y_{5}}, y_{5}$ is a path in $\bar{G}$. Since $Q$ is the longest path in $\bar{G}$, then $Q \backslash y_{1} \cup\left\{x_{1}, y\right\}$ is not a chordless path. Therefore $x_{1}$ has a neighbor (in $\bar{G}$ ) in $Q \backslash y_{1}$. Let $j$ be the lowest index such that $x_{1}$ is adjacent to $y_{j}$ in $\bar{G}$. Then $6 \leq j$ and $C=\left(x_{1}, y, y_{2}, Q_{y_{2} y_{j}}, y_{j}, x_{1}\right)$ is a big anti-hole in $G$, a contradiction. Hence $x_{1}$ is not adjacent to $y_{2}$, so $A \cup x_{1}$ is a co-connected set, $x_{1}$ is adjacent to $y_{3}, y_{4}$ and $y_{5}$ but not $y_{2}$, contradicting the maximality of $A$.

So every node in $A$ must have a neighbor in $P$. For every $y \in A$ let $h(y)$ be the minimum index such that $y$ is adjacent to $x_{h(y)}$, and let $h=\max _{y \in A} h(y)$. If $h>2$, then let $x \in A$ be such that $h=h(x)$ and let $H^{\prime}=\left(x, y_{3}, x_{1}, P_{x_{1} x_{h}}, x_{h}, x\right)$. $H^{\prime}$ is a big hole and $y_{5}$ is adjacent to $x$ and $y_{3}$ in $H^{\prime}$. Since $\left(H^{\prime}, y_{5}\right)$ is not a big cap, then $\left(H^{\prime}, y_{5}\right)$ must be a twin wheel, hence $y_{5}$ is adjacent to either $x_{1}$ or $x_{h}$. If $y_{5}$ is adjacent to $x_{1}$, then let $F$ be a shortest path from $y_{5}$ to $x_{h}$ in $V\left(P_{x_{h} x_{n}}\right) \cup\left\{y_{2}, y_{4}, y_{5}\right\}$, then $H^{\prime \prime}=\left(y_{5}, x_{1}, P_{x_{1} x_{h}}, x_{h}, F, y_{5}\right)$ is a big hole and $\left(H^{\prime \prime}, y_{3}\right)$ is a big cap. If $y_{5}$ is adjacent to $x_{h}$, then let $H^{\prime \prime}=H^{\prime} \cup y_{5} \backslash x$. Since, by definition of $h$, every node of $A$ has a neighbor in $P_{x_{1} x_{h}}$ and every node in $A$ is adjacent to $y_{3}$ and $y_{5}$, then $\left(H^{\prime \prime}, y\right)$ is a twin wheel for every $y \in A$. Since no node in $P$ is universal for $A$ and $A$ is co-connected, then there exists two nonadjacent nodes $u$ and $v$ in $A$ such that $u$ is adjacent to $x_{1}$ and not to $x_{h}$, and $v$ is adjacent to $x_{h}$ and not to $x_{1}$. Therefore $V(H) \cup\{u, v\} \backslash\left\{y_{5}\right\}$ induces a big cap, a contradiction. Therefore $h \leq 2$ and, since no node in $P$ is universal for $A, h=2$ and every node in $A$ is adjacent to $x_{1}$ or $x_{2}$. Since $x_{1}$ and $x_{2}$ are not universal for $A$ and $A$ is co-connected, there exists a chordless co-path $Z=z_{1}, \ldots, z_{k}$ contained in $A$ such that $z_{1}$ is adjacent to $x_{1}$ but not $x_{2}, z_{k}$ is adjacent to $x_{2}$ but not $x_{1}$ and all the intermediate nodes of $Z$ are adjacent to both $x_{1}$ and $x_{2}$. If $x_{2}$ is not adjacent to $y_{4}$, then $\left(y_{4}, x_{2}, z_{1}, Z, z_{k}, x_{1}, y_{4}\right)$ is a big anti-hole. Then $x_{2}$ is adjacent to $y_{4}$, so $\left(y_{4}, y_{3}, x_{2}, z_{1}, Z, z_{k}, x_{1}, y_{4}\right)$ is a big anti-hole, a contradiction.

\subsection{Meyniel graphs}

Lemmas 9, 10, 11 and 15 imply that, if $G$ and $\bar{G}$ do not contain a proper wheel or a long prism, then, if $G$ contains a cap, $G$ has a good skew-partition. Next we have to address the case in which $G$ does not contain any cap. Note that the class of Berge graphs containing no caps coincide with the class of Meyniel graphs, that is the class of graphs in which every odd cycle has at least 2 chords. Perfection of this class was proved by Meyniel [10], while Burlet and Fonlupt [2] showed that Meyniel graphs are either bipartite or can be decomposed by amalgams and clique cutsets. Hoàng [8], gave a short proof of a weaker result, namely:

Theorem 16 If $G$ is a Meyniel graph, then either $G$ is bipartite or $\bar{G}$ contains a starcutset or a U-cutset.

For the sake of completeness, we give a proof of Theorem 16, essentially following [8]. 
Proof: If $G$ is not bipartite, then, since $G$ is Berge, $G$ contains three pairwise adjacent nodes $u, v$ and $w$. Let $U$ and $V$ be, respectively, the set of neighbors of $u$ and $v$ in $\bar{G}$, and let $S$ be the connected component of $\bar{G} \backslash(U \cup V)$ containing $w$. Let $U^{\prime}, V^{\prime}$ and $X$ be, respectively, the set of nodes in $U \backslash V, V \backslash U$ and $U \cap V$ that are adjacent to some node of $S$ in $\bar{G}$. Note that, if $U^{\prime}=\emptyset$ or $V^{\prime}=\emptyset$, then $\{v\} \cup V^{\prime} \cup X$ or $\{u\} \cup U^{\prime} \cup X$, is a star cutset of $\bar{G}$ centered, respectively, at $v$ or $u$. Hence we may assume $U^{\prime} \neq \emptyset$ and $V^{\prime} \neq \emptyset$. Next we show that, in $\bar{G}$, every node in $U^{\prime}$ is adjacent to every node in $V^{\prime}$. Assume not and let $u^{\prime} \in U^{\prime}$ and $v^{\prime} \in V^{\prime}$ be nonadjacent in $\bar{G}$ and let $x_{u}$ and $x_{v}$ be, respectively, neighbors (in $\bar{G}$ ) of $u^{\prime}$ and $v^{\prime}$ in $S$ at minimum distance in $\bar{G}[S]$. Let $Q$ be a shortest path between $x_{u}$ and $x_{v}$ in $\bar{G}[S]$. Then $u, u^{\prime}, x_{u}, Q, x_{v}, v^{\prime}, v$ is a chordless path containing at least 5 nodes, hence $G\left[V(Q) \cup\left\{u, u^{\prime}, v, v^{\prime}\right\}\right]$ contains a small cap, a contradiction. If no connected component of $G\left[U^{\prime} \cup V^{\prime} \cup X\right]$ intersects both $U^{\prime}$ and $V^{\prime}$, then let $A$ be the union of all connected components of $G\left[U^{\prime} \cup V^{\prime} \cup X\right]$ intersecting $U^{\prime}$ and let $B=\left(U^{\prime} \cup V^{\prime} \cup X\right) \backslash A$. Then $A \cup B$ is a skew-cutset separating $S$ and $\{u, v\}$ in $\bar{G}, u$ is universal for $A$ while $v$ is universal for $B$, so $A \cup B$ is a U-cutset. Hence we can assume that there are nodes $u^{\prime} \in U^{\prime}$ and $v^{\prime} \in V^{\prime}$ such that there exists a chordless path $P$ between $u^{\prime}$ and $v^{\prime}$ in $G\left[X \cup\left\{u^{\prime}, v^{\prime}\right\}\right]$. Since, in $G, u^{\prime}$ is not adjacent to $v^{\prime}, P$ has length at least two, so $H=\left(v, u^{\prime}, P, v^{\prime}, u, v\right)$ is a big hole. $S$ is a co-connected set (in $G$ ) and, by definition of $U^{\prime}, V^{\prime}$ and $X$, no node in $P$ is universal for $S$ (in $G$ ). But then $S$ sees exactly one edge in $H$, namely $u v$. Since $G$ contains no caps, then every node in $S$ has a neighbor in $H \backslash\{u, v\}$, hence, for every $x \in S,(H, x)$ is a wheel which is not triangle-free. Note that every wheel which is not a twin wheel, a universal wheel or a triangle free wheel contains a cap. Therefore every node in $S$ is either the center of a twin wheel or of a universal wheel w.r.t. $H$. Also, there exist two nodes $x$ and $y$ in $S$ such that $(H, x)$ and $(H, y)$ are twin wheels and the only edge of $H$ that sees both $x$ and $y$ is $u v$. Assume $x$ and $y$ is a pair of nodes at minimum distance in $\bar{G}[S]$ with this property and let $Q$ be a shortest path in $\bar{G}[S]$ between $x$ and $y$. Assume $x$ is a twin of $u$ w.r.t. $H$ and $y$ is a twin of $v$ w.r.t. $H$. Let $u^{\prime}$ and $v^{\prime}$ be, respectively, the neighbors of $x$ and $y$ in $H \backslash\{u, v\}$. If $|Q|=1$, then $H^{\prime}=H \backslash u \cup x$ is a hole and $(H, y)$ is a cap. Hence $|Q| \geq 2$ and all the intermediate nodes of $Q$ are universal for $H$. $Q$ must have odd length, otherwise $C=\left(v^{\prime}, x, Q, y, u^{\prime}, v^{\prime}\right)$ is an odd anti-hole. But then, given $w \in H \backslash\left\{u, u^{\prime}, v, v^{\prime}\right\}, C^{\prime}=(w, x, Q, y, w)$ is an odd anti-hole, a contradiction

\section{References}

[1] C. Berge, Färbung von Graphen deren sämtliche bzw. deren ungerade Kreise starr sind (Zusammenfassung), Wissenschaftliche Zeitschrift, Martin Luther Universität Halle-Wittenberg, Mathematisch-Naturwissenschaftliche Reihe (1961) 114-115.

[2] M. Burlet and J. Fonlupt, Polynomial algorithm to recognize a Meyniel graph, Annals of Discrete Mathematics 21 (1984) 225-252. 
[3] M. Chudnovsky, N. Robertson, P. Seymour and R. Thomas, The strong perfect graph theorem (June 2002).

[4] V. Chvátal, Star-cutsets and perfect graphs, J. Combin. Theory B 39 (1985) 189199.

[5] M. Conforti and G. Cornuéjols, Graphs without odd holes, parachutes or proper wheels: a generalization of Meyniel graphs and of line graphs of bipartite graphs, $J$. Combin. Theory B 87 (2003) 300-330.

[6] M. Conforti, G. Cornuéjols and K. Vušković, Square-free perfect graphs (February 2001), to appear in J. Combin. Theory B.

[7] M. Conforti, G. Cornuéjols, K. Vušković and G. Zambelli, Decomposing Berge graphs containing proper wheels, preliminary draft, 2002.

[8] C. T. Hoàng, Some properties of minimal imperfect graphs, Discrete Math. 160 (1996) 165-175.

[9] L. Lovász, A characterization of perfect graphs, J. Combin. Theory B 13 (1972) 95-98.

[10] H. Meyniel, On the perfect graph conjecture, Discrete Mathematics 16 (1976) 339342.

[11] N. Robertson, P. Seymour and R. Thomas, presentation at the Workshop on Graph Colouring and Decomposition, Princeton, September 2001.

[12] F. Roussel and P. Rubio, About skew partitions in minimal imperfect graphs, $J$. Combin. Theory B 83 (2001), 171-190.

[13] D. B. West, Introduction to Graph Theory, Prentice Hall (1996). 